\title{
Bedeutet hohe Arbeitszufriedenheit, dass die Arbeitsbedingungen gut sind?
}

\author{
Reinhard Hofbauer ${ }^{\star}$ und Michael Schwingsmehl ${ }^{* *}$
}

\section{Zusammenfassung}

Die Qualität des Arbeitslebens ist eine wichtige Dimension der Lebensqualität und findet in vielen modernen Wohlfahrtsmaßen Berücksichtigung. Sowohl objektive als auch subjektive Indikatoren kommen bei der Messung der Arbeitsplatzqualität zur Anwendung. Anhand von Daten einer Bevölkerungsbefragung wird gezeigt, dass von hoher Arbeitszufriedenheit, die mittels Single-Item-Fragen gemessen wird, nicht einfach auf gute Arbeitsbedingungen geschlossen werden kann. Arbeitszufriedenheit erweist sich als komplexes Konstrukt, in dem Adaptions- und Vergleichsprozesse eine wichtige Rolle spielen.

Schlagwörter: Arbeitszufriedenheit, Arbeitsbedingungen, Adaption, Zufriedenheitsmessung, Anspruchsniveau

\section{Does a high level of job satisfaction reflect good working conditions?}

\section{Abstract}

Job satisfaction has proved to be an important dimension of individual well-being and is taken into account in many modern welfare indices. For the measurement of job satisfaction there are objective as well as subjective indicators in use. By means of data of a population survey it can be shown that high values in single item questions on job satisfaction alone do not suffice to indicate good working conditions. Individual job satisfaction has to be considered as a complex phenomenon which is strongly influenced by processes of adaption and comparison.

Keywords: job satisfaction, working conditions, adaption, aspiration level, measuring satisfaction

\footnotetext{
${ }^{*}$ Reinhard Hofbauer: Wissenschaftlicher Mitarbeiter am Zentrum für Zukunftsstudien der Fachhochschule Salzburg. E-Mail: reinhard.hofbauer@fh-salzburg.ac.at

${ }^{*}$ Michael Schwingsmehl: Wissenschaftlicher Mitarbeiter an der Wirtschaftsuniversität Wien, ehm. wissenschaftlicher Mitarbeiter am Zentrum für Zukunftsstudien der Fachhochschule Salzburg. E-Mail: michael.schwingsmehl@wu.ac.at
} 


\section{Einleitung}

Erwerbsarbeit nimmt nicht nur einen bedeutenden Teil der Lebenszeit ein und vermittelt die materiellen Voraussetzungen für hohe Lebensqualität, sie ist als wichtige Sozialisationsinstanz ein bedeutender gesellschaftlicher Integrationsfaktor und vermittelt über Beziehungen, Sinn und Anerkennung auch wichtige nichtmaterielle Beiträge zu hoher Lebenszufriedenheit. Zugleich ist Erwerbsarbeit ein knappes Gut. Die Zufriedenheit mit dem Arbeitsleben gilt daher als wichtige Determinante der gesamten Lebenszufriedenheit (Loscocco und \& Roschelle 1991; Argyle und Martin 1991). Der fünfte European Working Conditions Survey, der Indizes aus vier Subdimensionen der Arbeitsqualität (Einkommen, Erwartungen, Arbeitszeit und Arbeitsbedingungen) bildet, kommt zum Ergebnis, dass alle vier Subdimensionen mit subjektivem Wohlbefinden korrelieren, am stärksten die unmittelbaren Arbeitsbedingungen. Eine Steigerung der Arbeitsbedingungen um 10 Punkte erhöht, ceteris paribus, das subjektive Wohlbefinden um 3,5 Punkte (Eurofound 2012: 31).

Der hohe Stellenwert der Dimension der Arbeit kommt auch in breiten Lebensqualitäts- und Wohlfahrtsmaßen, die in den vergangenen Jahren entstanden sind, um gesellschaftliche Wohlfahrt und Lebensqualität besser zu messen, zum Ausdruck. Im Better-Life-Index der OECD wird Lebensqualität anhand von elf Indikatoren gemessen: mit „Work-LifeBalance“, „Education \& Skills“, „Income“ sowie „Jobs“ sind vier dieser elf Indikatoren arbeitsbezogen (OECD 2015). Die Eurostat Sponsorship Group on Measuring Progress, Well-being and Sustainable Development hat die Empfehlungen des Stiglitz-Sen-Fitoussi-Berichts zu zehn Schlüsselindikatoren der Lebensqualität operationalisiert. Mit „Material Living Conditions“, „Economic \& Physical Activity“, „Education“ und „Productive and valued activities“ haben vier von zehn Schlüsseldimensionen einen direkten Bezug zur Erwerbsarbeit (Eiffe et al. 2016). Im privaten Legatum Prosperity Index haben drei von acht Subindizes, aus denen ein Index für Wohlstand und Lebenszufriedenheit gebildet wird, einen engen Konnex zur Erwerbsarbeit (LegatumInstitute, 2015).

Das Interesse an der Arbeitsqualität speist sich nicht ausschließlich aus der Sorge um das menschliche Wohlbefinden. Gute Arbeitsbedingungen und hohe Arbeitszufriedenheit gelten angesichts der mannigfachen gegenwärtigen gesellschaftlichen und wirtschaftlichen Herausforderungen auch deshalb als unerlässlich, weil sie der besseren Ausschöpfung des Humankapitals dienen (Marrs 2010) und die vorrangigen EU-Ziele „[i]ntelligentes und nachhaltiges Wachstum ohne gute Jobs nicht zu haben sind“ (Holtgrewe 2013: 4).

So stehen nicht nur „mehr und bessere Arbeitsplätze“ (Europäischer Rat 200o) im Fokus der öffentlichen Aufmerksamkeit und hochrangiger politischer Initiativen, auch die Verbesserung der Arbeitsqualität, wie sie etwa im Strategischen Rahmen der EU für Gesundheit und Sicherheit am Arbeitsplatz 2014-2020 zum Ausdruck kommt, nimmt eine zentrale Rolle ein (Europäische Kommission 2016). Eines der sieben Ziele dieses strategischen Rahmens betrifft die Verbesserung der Erhebung statistischer Daten im Interesse einer besseren Informationsgrundlage und Weiterentwicklung von Monitoring-Instrumenten zur Erfassung der Arbeitsbedingungen.

\section{Wandel der Arbeitswelt}

Erwerbsarbeit ist seit geraumer Zeit einem beschleunigten Wandel unterworfen. Die Anforderungen an ArbeitnehmerInnen sind gestiegen und umfassen nicht mehr nur Arbeitsbereitschaft, Wissen oder anwendungsbezogene Qualifikationen, sondern auch ein hohes $\mathrm{Maß}$ an emotionaler Identifikation mit der Arbeitssituation. Auch die Arbeitsbedingungen haben sich verändert. Hierzu zählt etwa die Flexibilisierung der Arbeitsprozesse und der Arbeitszeiten, die Digitalisierung der Arbeitswelt, Entgrenzung und Verdichtung von Arbeit (Knobloch 2009), die wachsende Bedeutung von Wissens- und Interaktionsarbeit (Schmucker 2015), die Ausbreitung prekärer und befristeter Beschäftigungsverhältnisse und ein wachsendes Arbeitslosigkeitsrisiko.

Wurde in den 8oer- und 9oer-Jahren des vergangenen Jahrhunderts noch viel davon gesprochen, dass im Gefolge eines allgemeinen gesellschaftlichen Wertewandels (Inglehart 1977) Erwerbsarbeit ihre zentrale Stellung als sozialer Platzanzeiger verloren habe, immaterielle Aspekte vorherrschend geworden seien und sich eine normative Subjektivierung der Arbeit vollzogen habe (Baethge 1991), kann dieser Befund vor dem Hintergrund tiefgreifender sozialer und wirtschaftlicher Veränderungen als überholt gelten. In diesem Sinn stellten Eichmann und Saupe (2014: 296) auf der Grundlage eines Langfristvergleichs der Daten der European Values Studies fest, dass so gut wie alle beruflichen Ziele von ArbeitnehmerInnen wichtiger geworden sind: Materielle (extrinsische) Gratifikation, 
intrinsische Leistungsbedürfnisse sowie soziale Kontaktbedürfnisse haben an Bedeutung gewonnen.

Begleitet werden diese sozialen Veränderungen von einer tiefgreifenden allgemeinen gesellschaftlichen Verunsicherung, die sich nicht nur in strukturellen Problemgruppen wie bei Geringqualifizierten oder bildungsfernen Schichten, sondern auch in gesicherten Beschäftigungssituationen und in finanziell abgesicherten Haushalten zeigt. Wahrgenommene Verunsicherung bedarf offenbar keiner eigenen Gefährdungslage. Lengfeld und Hirschle sprechen in diesem Zusammenhang von einem Spill-over-Effekt der Diffusion von Unsicherheit ausgehend von akut Gefährdeten in breite Kreise der Bevölkerung (Lengfeld/Hirschle 2009).

Angesichts dieser Diagnosen ist es erstaunlich, dass Messungen zur Arbeitszufriedenheit nach wie vor sehr hohe durchschnittliche Zufriedenheit zeigen und zwar weitgehend unabhängig davon, wie diese gemessen werden.

\section{Erkenntnisse der Arbeitszufriedenheitsforschung}

Die Erforschung der Arbeitszufriedenheit hat eine lange Tradition und ist stärker anwendungsorientiert als die Lebensqualitätsforschung. Dies vor allem deshalb, weil Arbeitszufriedenheit auch als wichtiger Prädiktor der Arbeitsproduktivität gilt, während es die Erforschung der Lebenszufriedenheit immer mit den Bedingungen des guten Lebens für den Einzelnen oder die Gesellschaft zu tun hat und institutionelle Interessen allenfalls eine vergleichsweise hintergründige Rolle spielen. Die Diskussion um die Arbeitszufriedenheit aus arbeitswissenschaftlicher Perspektive konzentrierte sich häufig auf die Frage, wie Unternehmen die Arbeitszufriedenheit ihrer MitarbeiterInnen verbessern können. Dementsprechend finden sich in der empirischen Organisationspsychologie neben Leistungsindizes häufig Kriterien der Arbeitszufriedenheit (Rosenstiel 2003). ${ }^{1}$

Umfassende Untersuchungen zur Qualität der Arbeit und der Arbeitszufriedenheit fokussieren vor

1 Ein eindeutig hoher Zusammenhang zwischen Arbeitszufriedenheit und Arbeitsleistung konnte bis dato aber nicht zweifelsfrei belegt werden. Judge et al. errechneten im Rahmen einer Metastudie aus 312 unabhängigen Stichproben einen mittleren Zusammenhang von $\mathrm{r}=, 30$ und einer deutlich niedrigeren unkorrigierten Korrelation. Die Autoren bezeichnen diese mittlere Korrelation als "moderate in magnitude and distinguishable from zero" (Judge et al. 2001: $385)$. allem auf die Arbeitsbedingungen. Van Saane et al. ermittelten in einer Metastudie über 35 Arbeitszufriedenheitsuntersuchungen folgende relevante Aspekte der Arbeitszufriedenheit: Arbeitsinhalt, Autonomie, Entwicklungsmöglichkeiten, Einkommen, Unterstützung, Kommunikation, Mitarbeiterbeziehungen, Sinngehalt und Arbeitsbelastungen (Van Saane et al. 2003).

Der European Working Conditions Survey nennt als wichtigste Einflussgrößen neben dem Einkommen die Lage der Arbeitszeit (keine Schicht- oder Nachtarbeit), keine Arbeit in ermüdender oder schmerzhafter Haltung oder unter Zeitdruck, gute Vereinbarkeit von Berufs- und Privatleben, hoher Ermessensspielraum und hohe soziale Unterstützung bei der Arbeit, Führungsverhalten, gute Karriereaussichten und Sicherheit des Arbeitsplatzes (Eurofound 2014). Auf Basis der Analyse langjähriger Daten des österreichischen Arbeitsklimaindex stellt Raml fest: „[...] die wichtigsten Punkte für die Arbeitszufriedenheit sind das Betriebsklima, das Verhältnis zum Vorgesetzten und den Arbeitskollegen, die ergonomischen Belastungen, die psychischen Überforderungen am Arbeitsplatz wie Zeitdruck und Verantwortung, die Unterbrechungen des Arbeitsvollzugs und Gratifikationskrisen" (Raml 2012: 19). Diese wenigen Beispiele zeigen, dass es in Abhängigkeit der jeweiligen Konzepte guter Arbeit verschiedene Indikatoren, Messverfahren und Ergebnisse gibt.

Weitergehende Einigkeit zeigt die empirische Arbeitszufriedenheitsforschung bei soziodemografischen Differenzen der Arbeitszufriedenheit, vor allem zwischen Berufs- sowie Einkommensgruppen und nach dem Bildungsstand. Besserausgebildete mit höheren Einkommen weisen höhere Zufriedenheitswerte auf, vor allem untere Einkommen dagegen unterdurchschnittliche Zufriedenheitswerte (Eurofound 2014; Statistik Austria 2015).

Der seit den 1990er-Jahren regelmäßig erhobene Arbeitsklimaindex, der Bewertungen verschiedener Dimensionen der Arbeitsqualität durch betroffene ArbeitnehmerInnen zusammenfasst, weist eine große Bandbreite auf: von 118 Punkten bei Büroangestellten ohne KundInnenkontakt bis hin zu 87 Punkten bei LeiharbeiterInnen. Unterschiede bei der Bewertung der Erwerbsarbeit entsprechen offenbar bis zu einem gewissen Ausmaß unterschiedlichen Beschäftigungsund Arbeitsbedingungen. Eichmann \& Saupe schlussfolgern, „[...] dass die Befragten ihre Arbeit durchaus ,realistisch" wahrnehmen" (Eichmann und Saupe 2014: 315). Die berichteten soziodemografischen Unter- 
schiede ändern jedoch kaum etwas an einer hohen durchschnittlichen Zufriedenheit mit den Arbeitsbedingungen.

Neben unterschiedlichen Aspekten der konkreten Arbeitssituation und des Arbeitsumfeldes üben „Moderatorvariablen“, den tatsächlichen Bewertungen der Arbeitsbedingungen vorgelagert, Einfluss auf die Arbeitszufriedenheit der Beschäftigten aus. Zu diesen zählen biografische sowie Persönlichkeitsmerkmale, wobei letzteren von ForscherInnen unterschiedlich hohe Bedeutung beigemessen wird und Arbeitszufriedenheit zuweilen als Folge von individuellen, relativ unbeeinflussbaren Persönlichkeitsmerkmalen gesehen wird (Hölzl 2011). Fietze, der auf Basis des deutschen Sozio-oekonomischen Panels (SOEP) den Einfluss von Arbeitsbedingungen und Persönlichkeitseigenschaften sowie ihrer Interaktion untersucht hat, kam zu dem Ergebnis: „Individuelle Arbeitsbedingungen (insb. eine geringe Effort-Reward-Imbalance und hohe Autonomie) und Persönlichkeit (insb. emotionale Stabilität) spielen eine entscheidende Rolle, um höhere Arbeitszufriedenheit zu erreichen, aber: Die höchste Erklärungskraft haben die Arbeitsbedingungen (EffortReward-Imbalance und Autonomie)“"(Fietze 2011: 2). In dieselbe Richtung deuten die Ergebnisse von Abele et al.: „Arbeitszufriedenheit [...] beruht vielmehr auf mehr oder weniger positiven Wahrnehmungen der eigenen Arbeitsbedingungen" (Abele et al 2006: 225).

Gute objektive Arbeitsbedingungen bilden offenbar die Grundlage der Arbeitszufriedenheit, das konkrete Zufriedenheitsurteil der Beschäftigten ist allerdings durch biografische und komplexe innerpsychische Prozesse, bei denen Ansprüche, Erwartungen, Vergleiche an die Situation bzw. mit anderen Situationen eine wichtige Rolle spielen, moderiert.

\section{Theorien der Arbeitszufriedenheit}

Das Konstrukt „Arbeitszufriedenheit“ steht seit Jahrzehnten im Mittelpunkt kritischer Auseinandersetzungen von PsychologInnen und SozialwissenschaftlerInnen, wobei die Vorstellungen unterschiedlicher AutorInnen zur Entstehung der Arbeitszufriedenheit teils weit auseinandergehen. „Arbeitsqualität“ , „Beschäftigungsqualität“, „Arbeits- und Beschäftigungsqualität“, „faire Beschäftigung“, „menschenwürdige Arbeit“ - es gibt verschiedene Bezeichnungen für Konzepte, um die Qualität der Arbeit im weitesten Sinne zu messen.

In der engeren Arbeitszufriedenheitsforschung wurden verschiedene Konzeptionen entwickelt:
Bedürfnistheoretische Konzeptionen konzipierten Zufriedenheit als Bedürfnisbefriedigung, anreiztheoretische Zugänge betonten stärker die äußeren Aspekte der Arbeitssituation und ihrer Bewertung. Bei kognitiven Ansätzen entwickelt sich Arbeitszufriedenheit durch den Vergleich der Erwartungen an die Arbeitssituation („Soll“) und eines subjektiv wahrgenommenen „Ist-Zustands“. Einflussreiche Theorien der Arbeitszufriedenheit - also des Zusammenhangs von Arbeitsbedingungen und subjektivem Wohlbefinden - sind etwa das Anforderungs-Kontroll-Modell (job demand-control model „DC-model“) und das Effort-Reward-Imbalance-Modell (ERI-Modell). Das DC-Modell nennt zwei Parameter zur Bestimmung von Belastung und Beanspruchung von ArbeitnehmerInnen: die Arbeitsanforderungen und der Handlungsspielraum bzw. die Autonomie am Arbeitsplatz. Beide können sowohl in geringer als auch in hoher Ausprägung vorliegen. Das DC-Modell geht davon aus, dass starke Belastungen am Arbeitsplatz entstehen, wenn die Arbeitsanforderungen hoch sind, aber den Arbeitenden aufgrund von fehlender Autonomie nur ein geringer Handlungsspielraum zur Verfügung steht. Kommt aufgrund fehlender Autonomie keine Handlung zustande, geht die Handlungsenergie als mental strain in Stresssymptome über (Bakker/ Demerouti 2007). Im Effort-Reward-Imbalance-Modell von Siegrist kommt es zu Gratifikationskrisen, wenn die Balance zwischen Arbeitseinsatz und entsprechender Belohnung nicht ausgeglichen ist. Gratifikationskrisen können folglich zu psychischer Belastung und langfristig zu psychischen Erkrankungen führen. Einsatz und Anstrengungen müssen Gratifikationen in einem ausgeglichenen Verhältnis gegenüberstehen, damit ArbeitnehmerInnen dauerhaft motiviert sind und keine gesundheitlichen Folgeschäden erleiden. Gratifikationen können zum Beispiel in Form von Wertschätzung, Bezahlung oder Einflussmöglichkeiten erfolgen (Siegrist 2000). Im Fokus des Modells stehen damit nicht nur Belastungen am Arbeitsplatz, sondern auch die Erreichbarkeit entsprechender Gratifikationen.

Das Modell der Qualitäten der Arbeitszufriedenheit von Bruggemann (1975) basiert nicht auf der Analyse der Gesamtarbeitszufriedenheit, sondern betrachtet verschiedene einzelne Qualitäten der Zufriedenheit. In diesem Modell wird das Verhältnis zwischen Person und Situation als dynamisch verstanden: Veränderungen der Arbeitssituation durch die Person in Abhängigkeit von ihren situativen Kontroll- und Einflussmöglichkeiten werden ebenso berücksichtigt 
wie Veränderungen der Ziele und des Anspruchsniveaus durch die Person. In diesem Licht ist Arbeitszufriedenheit nicht ein Produkt, sondern Abbild eines Prozesses: Es geht nicht um Quantitäten, sondern um Qualitäten von Zufriedenheit. Welche Form der Arbeitszufriedenheit auftritt, ist zunächst von einem individuellen Vergleich zwischen den eigenen Bedürfnissen und Erwartungen einerseits (= SOLL) und den Möglichkeiten ihrer Realisierung in der jeweiligen Arbeit andererseits (= IST) abhängig. Je nach Ausgang des Soll-Ist-Vergleichs und des Anspruchsniveaus ergeben sich dann die unterschiedlichen Formen der Arbeitszufriedenheit bzw. -unzufriedenheit. Bruggemann unterscheidet „Progressive Arbeitszufriedenheit", bei der der Soll-Ist-Vergleich positiv ist und das Anspruchsniveau dadurch steigt. Bei „Stabilisierter Arbeitszufriedenheit“" ist der Soll-Ist-Vergleich positiv und das Anspruchsniveau bleibt stabil. Bei „Resignativer Arbeitszufriedenheit" ist der Soll-Ist-Vergleich negativ und zur Kompensation sinkt das Anspruchsniveau. Weitere Formen der Arbeitszufriedenheit sind "Pseudo-Zufriedenheit“ mit einem negativen SollIst-Vergleich aber unverändertem Anspruchsniveau, dafür wird die Situation verfälscht (geschönt) wahrgenommen. Bei „Konstruktiver Arbeitszufriedenheit“ fällt der Soll-Ist-Vergleich ebenfalls negativ aus, das Anspruchsniveau bleibt unverändert, aber man arbeitet an Lösungsversuchen (Bruggemann 1976).

\section{Die Messung der Arbeitszufriedenheit}

Über die Qualität der Arbeit wird heute in vielen, unterschiedlich angelegten Untersuchungen berichtet. Diese Untersuchungen greifen diverse Theorieelemente guter Arbeit auf. Aus unterschiedlichen Modellen der Arbeitszufriedenheit folgt auch, dass gute Arbeit und hohe Arbeitszufriedenheit unterschiedlich gemessen werden. In der Praxis sind zwei Arten der Erfassung von Arbeitszufriedenheit üblich: Zum einen wird die Qualität von Merkmalen der Arbeit (Dauer, Lage und Flexibilität der Arbeitszeit, Erwerbseinkommen, physische Arbeitsbedingungen wie Lärm oder Schmutz, Freude am Arbeitsergebnis, Betriebsklima etc.) erfasst und manchmal anschließend $\mathrm{zu}$ einem Gesamtbild verdichtet. $\mathrm{Zu}$ den wichtigsten Quellen dieses Typs der arbeitsqualitätsbezogenen empirischen Forschung zählen der europaweit durchgeführte Working Conditions Survey (Eurofound), das Decent-Work-Konzept der ILO, für Österreich vor allem die Arbeitskräfteerhebung der Statistik Austria sowie der Arbeitsklimain- dex der Arbeiterkammer. Der Arbeitsklimaindex etwa misst die Arbeitszufriedenheit über eine normierte Indexbildung auf Basis von ca. 25 Bewertungen zur eigenen Arbeitssituation. Dabei wird einerseits ein Gesamtindikator („Arbeitsklima-Index“) ermittelt, darüber hinaus aber auch einzelne Teilindikatoren (zu Arbeit, Betrieb, Gesellschaft, Erwartungen). Der sechste European Working Conditions Survey (EWCS) misst die Arbeitsqualität in sieben Dimensionen (physische Arbeitssituation, Intensität der Arbeit, Arbeitszeit, Einkommen, Erwartungen, Qualifikationen und soziales Arbeitsumfeld) und überprüft die Dimensionen hinsichtlich ihrer Korrelation mit subjektiver Zufriedenheit, die mit den $\mathrm{WHO}-5$-Kriterien gemessen werden.

Die objektiven Indikatoren, anhand derer Arbeitsqualität gemessen wird, unterscheiden sich teilweise. Häufig wird für sie auch in Anspruch genommen, dass sie als empirisch ermittelte Korrelate der Arbeitsund Lebensqualität gelten (Eurofound 2012). Solche objektiven Zugänge basieren letztlich auf einer Theorie menschlicher Bedürfnisse. Aus der Beschreibung und Bewertung der beobachtbaren Umstände der Arbeit wird auf die Arbeitsqualität als Fähigkeit durchschnittliche menschliche Bedürfnisse $\mathrm{zu}$ befriedigen, geschlossen. Der EWCS folgt der Auffassung, dass nur ein „objektiver“ Zugang zur Untersuchung von Arbeitsqualität verteidigt werden kann. Denn subjektive Wohlbefindensmaße korrespondieren nicht notwendigerweise mit der Befriedigung von Bedürfnissen und sind daher als Proxys für die Arbeitsqualität ungeeignet, weil Faktoren wie Erwartungen oder Persönlichkeitsmerkmale die subjektive Arbeitszufriedenheit beeinflussen (Eurofound 2012: 10). Diese objektiven Zugänge lassen sich auch theoretisch erweitern. So hat Green (Green 2006) Sens Capability Approach adaptiert: ein guter Job eröffnet ArbeitnehmerInnen eine hohe „capability“, Dinge zu tun, die sie schätzen. Demnach ist die Fähigkeit, Wohlbefinden zu erreichen, abhängig davon, inwieweit Beschäftigte in die Lage versetzt werden, Einfluss über ihre Arbeit auszuüben, um ihre persönlichen arbeitsbezogenen Ziele zu erreichen. Während persönliche Bedürfnisse variieren können, zeichnet sich ein guter Job dadurch aus, dass er die volle Bandbreite solcher Bedürfnisse befriedigen kann. Das ILO-Konzept von „decent work“ steht in dieser Tradition, wenn es auch eine größere Bandbreite von Bedürfnissen adressiert. Soweit Zufriedenheit aus Bedürfnisbefriedigung resultiert, ist dieses Vorgehen plausibel. 
Der Versuch, anhand äußerer Arbeitsplatzmerkmale die Qualität der Arbeit und der Arbeitszufriedenheit $\mathrm{zu}$ bestimmen, unterscheidet sich grundsätzlich von der direkten Messung der Arbeitszufriedenheit. Die direkte Messung der subjektiven Arbeitszufriedenheit erfolgt in aller Regel durch die Frage „Wie zufrieden sind Sie mit Ihrer beruflichen Tätigkeit", wobei die Befragten auf einer Skala von o bis 10 Bewertungen abgeben können. Die direkte Messung der Arbeitszufriedenheit bringt damit die subjektive Komponente des Arbeitslebens ins Spiel. Nicht die Arbeitsumstände selbst, sondern die darauf bezogenen Bewertungen der Beschäftigten stehen im Interesse der Messung. Hinter diesem Zugang zur Messung der Arbeitsqualität steht die Auffassung, dass der subjektive Nutzen, den Beschäftigte aus ihren Arbeitsverhältnissen ziehen, unterschiedlich ausfallen kann. So können zwei Personen dieselben Tätigkeiten unter identen Rahmenbedingungen ausüben, aber in unterschiedlichem Ausmaß zufrieden sein. Es kann bei geringer Arbeitszufriedenheit eine gute Arbeitsqualität („Unzufriedenheitsdilemma") oder trotz schlechter Arbeitsbedingungen hohe Zufriedenheit („Zufriedenheitsparadox“) vorliegen (Zapf 1972).

Selbstangaben der Arbeits- oder Lebenszufriedenheit gelten als reliabel und valid (Eid/Larsen 2008; Veenhoven 2002). Wanous und Reichers berichten im Rahmen ihrer Metastudie zur Arbeitszufriedenheit einen hohen Korrelationskoeffizienten von ,67 zwischen Single-Item-Messungen der Arbeitszufriedenheit und Multi-Item-Messungen (Wanous et al. 1997: 250). Dennoch gilt die Befragung von Zufriedenheiten als nicht unproblematisch. Sie ist weder eine verifizierbare Erfahrung, noch eine bekannte Tatsache wie das Alter. „Es ist ein globales retrospektives Urteil, das in den meisten Fällen nur dann konstruiert wird, wenn man danach gefragt wird“ (Kahneman et al. 2004: 1177). Die in Single-Item-Befragungen übliche Frage zur allgemeinen Arbeitszufriedenheit ruft reflektierte Antworten hervor, womit auch gesellschaftliche Vorstellungen eines guten Lebens in die Beurteilung mit einfließen und stärker sozial erwünschte Antworten hervorgerufen werden. Dagegen bleiben emotionale Aspekte weitgehend unberücksichtigt. So bleibt fraglich, ob das Konstrukt Arbeitszufriedenheit hinreichend erfasst wird. Denn so wie die jüngere Well-Being-Forschung subjektives Wohlbefinden als komplexes Konstrukt aus affektiven und kognitiven Komponenten versteht (Diener et al. 1999), definiert die Arbeitspsychologie Arbeitszufriedenheit als emotionale und kognitive
Reaktion auf die jeweilige Arbeitssituation (Six/Felfe 2004). Kahneman und Deaton benennen diese Unterscheidung, indem sie zwischen Lebensbewertung und emotionalem Wohlbefinden unterscheiden (Kahneman/Deaton 2010). Letzteres bezieht sich auf die Qualität alltäglicher Erfahrungen, etwa wie erfüllend oder frustrierend die Zeit während der Arbeit empfunden wird - ein Aspekt, der bei einer lediglich stark kognitiv ausgerichteten Frage nach der Arbeitszufriedenheit unberücksichtigt bleibt. Eine Reihe von Messungen der Arbeitszufriedenheit folgt diesem Vorgehen: So erfasst die Statistik-Austria-Initiative die Arbeitszufriedenheit durch die Frage an die Erwerbstätigen „Wie zufrieden sind Sie mit Ihrer Arbeit?“ Fast 90 \% wählten einen Wert oberhalb der Mitte (5), nur $4 \%$ einen Wert darunter. $24 \%$ gaben an, mit ihrer Arbeit vollkommen zufrieden zu sein. Die mittlere Arbeitszufriedenheit lag bei einem Wert von 8,o (Statistik Austria 2014). Zu ähnlich hohen Werten kommt Brenke in einer DIW-Studie zur Arbeitszufriedenheit in Deutschland mit Daten aus dem Sozio-oekonomischen Panel (SOEP) nach demselben Erhebungsverfahren. „Die große Mehrzahl der Beschäftigten in Deutschland ist mit ihrer Arbeit zufrieden. An diesem Bild hat sich in den vergangenen 20 Jahren nichts verändert. Auf der von null (= sehr unzufrieden) bis zehn (= sehr zufrieden) reichenden Skala konzentrieren sich die Angaben auf die Werte von sieben bis neun. Nur jeder achte Erwerbstätige gibt Skalenwerte von null bis vier an, ist also mit dem Job eher unzufrieden. Der Mittelwert der Arbeitszufriedenheit liegt bei 7,1, und mit Blick auf sozioökonomische Merkmale zeigen sich keine nennenswerten Unterschiede“ (Brenke 2015: 718).

Dass Befragungen zur Arbeitszufriedenheit über Jahrzehnte ein konstantes Niveau von 70 bis $80 \%$ an Zufriedenen oder sehr Zufriedenen zeigen, hat wiederholt Anlass zu Zweifeln und Kritik gegeben - vor allem an der Methodik der Befragungen. Kritisiert wurde vor allem, dass eine hohe Arbeitszufriedenheit in einer Arbeitsgesellschaft, in der Erwerbsarbeit gleichermaßen Sinnressource, Statusanzeiger und zugleich ein knappes Gut ist, in hohem Ausmaß sozial erwünscht ist (Bolder/Schulte: 2005), veränderte Ansprüche und Erwartungen nicht entsprechend berücksichtigt werden und damit Messungen der Arbeitszufriedenheit auch die Bedeutung jener Werte zeigen, die der Arbeit in der jeweiligen gesellschaftlichen Situation gerade zukommen (Hardering 2011). An der Frage, ob mit einem single-item das Konstrukt Arbeitszufriedenheit hinreichend erfasst wird - ob es den Beschäftig- 
Tabelle 1: Einfluss der Arbeitszufriedenheit auf die Lebenszufriedenheit

\begin{tabular}{|c|c|c|c|c|c|}
\hline \multicolumn{6}{|c|}{ Wie zufrieden sind Sie insgesamt mit Ihrer beruflichen Tätigkeit? } \\
\hline niedrig & mittel & hoch & MW (1-3) & Std. Abw. & $\mathrm{N}$ \\
\hline 15,3 & 52,4 & 32,3 & 2,2 & 0,7 & 548 \\
\hline
\end{tabular}

Anmerkung: Gültige Prozent

ten also auch tatsächlich gut geht, wenn sie eine hohe Arbeitszufriedenheit bekunden -, setzt die nachfolgende Untersuchung an.

\section{Arbeitszufriedenheit im Bundesland Salzburg}

Die nachfolgenden Ausführungen basieren auf einer Bevölkerungsbefragung zu Fragen der Lebensqualität, die am Zentrum für Zukunftsstudien der FH Salzburg im Rahmen des Forschungsprojekts „Lebensqualität und Innovation im Bundesland Salzburg" entworfen und im Zeitraum Dezember 2015 bis Februar 2016 mittels standardisierten telefonischen Interviews vom Institut für empirische Sozialforschung (IFES) durchgeführt wurde. Dabei wurden 842 vollständige Interviews mit erwachsenen Beschäftigten ( $a b 18$ Jahren) im Bundesland Salzburg und mit 705 Personen in Österreich (ohne Salzburg) geführt. ${ }^{2}$ Zunächst wurden den Beschäftigten Fragen nach der allgemeinen Lebensund Arbeitszufriedenheit vorgelegt. Bei der Frage „Wie zufrieden sind Sie insgesamt mit Ihrer beruflichen

2 Die Stichprobe wurde vorrangig auf Grundlage der öffentlichen Telefonverzeichnisse gezogen. Hier wurden 842 Interviews realisiert, zusätzlich wurde das RLD-Verfahren („random last digit“) angewendet. Dabei werden durch Variation der Endziffern Zufallstelefonnummern generiert, um auch Geheimnummern zu erreichen. Die Berücksichtigung von Festnetz- und Mobilnetznummern in der Stichprobe erfolgt im Verhältnis des Marktanteils. Bei Festnetznummern im Haushalt kam ein Zufallsverfahren zur Auswahl der zu befragenden Zielperson (Geburtstagsregel) zur Anwendung. In der Summe wurde ca. die Hälfte der Interviews mittels RLD-Verfahren realisiert. Zur Erhöhung der Ausschöpfung erfolgten einerseits Terminvereinbarungen, andererseits bis $\mathrm{zu}$ drei Kontaktversuche zu unterschiedlichen Tageszeiten über mehrere Wochen verteilt.

Die Bruttostichprobe betrug 7.468, es wurden 1.547 vollständige Interviews geführt (Nettostichprobe), das entspricht einer Ausschöpfung von $21 \%$. Gewichtet wurden Geschlecht, Alter, Schulbildung getrennt für Salzburg und Nicht-Salzburg. Bei letzterer Gruppe wurden zudem die Relationen der Bundesländer (S) zueinander korrekturgewichtet. Die Fallzahlen wurden zudem auf $n=800(S)$ und $n=700$ (Rest-Ö) hinunterprojiziert. Die Fragen wurden randomisiert.
Tätigkeit? “3 liegt der Mittelwert auf einer Skala von o bis 10 bei 7,5 und damit sogar etwas höher als bei der Frage nach der Zufriedenheit mit der Lebensqualität (MW: 7,1). Diese hohen Werte der Arbeitszufriedenheit bestätigen im Wesentlichen die Ergebnisse vergleichbarer Befragungen nach der Arbeitszufriedenheit, wie sie etwa im Arbeitsklimaindex, im Rahmen von EU-SILC und auch im European Working Conditions Survey berichtet werden. Etwa 15 \% der befragten Erwerbstätigen zeigten ein geringes Niveau an Zufriedenheit mit ihrer beruflichen Tätigkeit. Mehr als doppelt so viele Erwerbstätige (32,3 \%) wiesen ein hohes, die Mehrheit $(52,4 \%)$ jedoch ein mittleres Zufriedenheitsniveau auf. ${ }^{4}$

Hinsichtlich des Geschlechts zeigen sich keine signifikanten Mittelwertunterschiede, ${ }^{5}$ ebenso wenig hinsichtlich der höchsten abgeschlossenen Ausbildung $^{6}$ oder der Gemeindegröße (bis und über 50.000 EinwohnerInnen $)^{7}$. Dagegen sind ältere Erwerbstätige $(45+)$ etwas zufriedener (MW: 7,8) als jüngere (MW: $7,3) .{ }^{8}$ Nennenswerte Unterschiede zeigen sich bei der beruflichen Stellung: ArbeiterInnen (MW: 7,1) und Angestellte (MW: 7,4) sind weniger zufrieden mit ihrer beruflichen Situation als öffentlich Bedienstete (MW: $7,8)$.

6.1 Wie hoch ist der Einfluss der Arbeitszufriedenheit auf die Lebenszufriedenheit?

Ein signifikanter und direkter Zusammenhang zwischen der Zufriedenheit im Beruf und der allgemeinen Lebenszufriedenheit ist wiederholt festgestellt

3 Befragt wurden hier nur Selbstständige, Unselbstständige, Lehrlinge im Alter zwischen 20 und 65 Jahren.

4 Dem Vorgehen von Eurostat folgend, wurde die von o bis 10 gehende Zustimmungs-Skala ex-post in die Kategorien niedrige (0-5), mittlere (6-8) und hohe (9-10) Zufriedenheit transformiert. Dies nicht nur zur übersichtlicheren Darstellung der Daten, auch wird dadurch ein Vergleich mit den von Eurostat präsentierten Ergebnissen der EU-SILCErhebung ermöglicht (siehe Eurostat 2015).

5 Mittelwertdifferenz: -0,135, Sig. (2-seitig): 0,439.

6 F-Wert ANOVA-Test: 1,009, Sig.: 0,411.

7 Mittelwertdifferenz: 0,28, Sig. (2-seitig): o,186.

8 Mittelwertdifferenz: -0,424, Sig. (2-seitig): o,o18. 
Abbildung 1: Linearität des Zusammenhangs

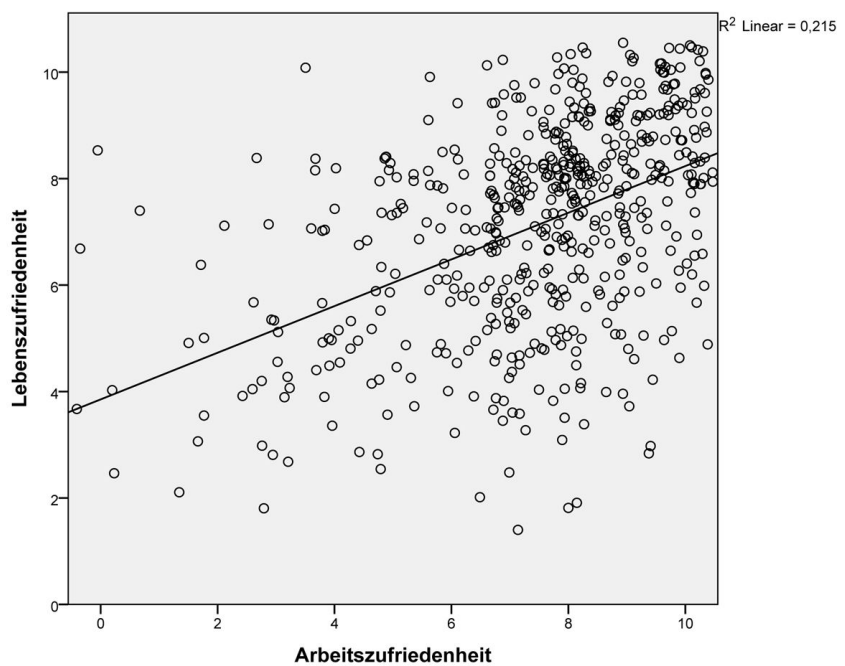

Anmerkung: Zur Darstellung der überlappenden Datenpunkte wurde die jitter-Funktion herangezogen, die die Werte der beiden Vektoren um einen kleinen Zufallsbetrag verändert.

Tabelle 2: Einfluss der Arbeitszufriedenheit auf die Lebenszufriedenheit

\begin{tabular}{cccc}
\hline & \multicolumn{3}{c}{ Modellzusammenfassung } \\
\hline $\mathrm{F}$ & $\mathrm{p}$ & $\mathrm{R}^{2}$ & $\mathrm{~N}$ \\
148,837 & $<, 001$ &, 215 & 548 \\
& Lebenszufriedenheit $=\alpha+\beta_{1}$ Arbeitszufriedenheit $_{\mathrm{t}}+\varepsilon_{\mathrm{t}}$ & $\mathrm{T}$ & $\mathrm{p}$ \\
Prädiktor & Nichtstand. Reg. Koeffiz. & 13,803 & $\mathrm{p}<, 001$ \\
Konstante & 3,852 & 12,200 & $\mathrm{p}<, 001$ \\
Arbeitszufriedenheit & 0,438 & &
\end{tabular}

Anmerkung: Vollständige Ergebnisauflistung siehe Anhang: Regression 1

worden. Wer mit seiner beruflichen Tätigkeit zufrieden ist, ist auch mit seinem Leben insgesamt zufriedener und umgekehrt (Argyle/Martin 1991; Poggi/Villosio 2015). Da Lebenszufriedenheit aber mit der Zufriedenheit in bestimmten Lebensbereichen stark korreliert, ist ein solcher Zusammenhang nicht überraschend (Eiffe et al. 2016: 17). Nichtsdestotrotz ist ein Blick auf die Einflussstärken diverser Einflussfaktoren auf die allgemeine Lebenszufriedenheit interessant. Da (wie in Abbildung 1 geprüft) die Linearität des Zusammenhangs gegeben ist, wird zunächst der Einfluss der allgemeinen Arbeitszufriedenheit (gemessen durch die Variable: „Wie zufrieden sind Sie insgesamt mit Ihrer Arbeitssituation") auf die allgemeine Lebenszufriedenheit anhand des Modells

Lebenszufriedenheit $_{t}=\alpha+\beta_{1}$ Arbeitszufriedenheit $_{t}+\varepsilon_{t}$

mit einer einfachen linearen Regression geschätzt. Das Ergebnis der Regressionsschätzung (Tabelle 2) zeigt, dass rd. 22 \% der Varianz der Lebenszufriedenheit durch das Maß der Zufriedenheit mit der Arbeitssituation erklärt werden kann. ${ }^{9}$

9 Streng genommen weisen die 10-teiligen Selbsteinschätzungs-Skalen für Lebensqualität und Arbeitszufriedenheit jeweils ein ordinales Skalenniveau auf. Eine Schätzung mittels OLS setzt jedoch eine metrische abhängige Variable voraus. Dementsprechend wäre eine für ordinale Daten geeignete Schätzmethode (wie etwa eine Probit-Analyse) der OLS-Schätzung vorzuziehen. An dieser Stelle sei jedoch etwa auf Ferrer-i Carbonell und Frijters (2004) oder Boarini et al. (2012) verwiesen, die zeigen, dass im vorliegenden Fall sowohl eine OLS-Schätzung als auch Schätzmethoden für ordinale Daten zu sehr ähnlichen Ergebnissen führen. Auch der Nagelkerke-Test für ordinale Regressionen bestätigt den hohen Erklärungsanteil der Arbeitszufriedenheit für die Lebenszufriedenheit: Das Pseudo- $\mathrm{R}^{2}$ liegt mit $24 \%$ ebenfalls sehr nahe an dem Ergebnis der OLS-Schätzung. Aufgrund der einfacheren und intuitiveren Interpretation von OLSSchätzern wird den Selbsteinschätzungs-Skalen deshalb ein metrisches Skalenniveau zugeschrieben und eine OLS-Schätzung durchgeführt. 
Tabelle 3: Einflussgrößen auf die allgemeine Lebensqualität

\begin{tabular}{|c|c|c|c|}
\hline \multicolumn{4}{|c|}{ Modellzusammenfassung } \\
\hline $\mathrm{F}$ & $\mathrm{p}$ & Korr. $\mathrm{R}^{2}$ & $\mathrm{~N}$ \\
\hline 26,245 & $<, 001$ & 0,372 & 383 \\
\hline \multicolumn{4}{|c|}{ 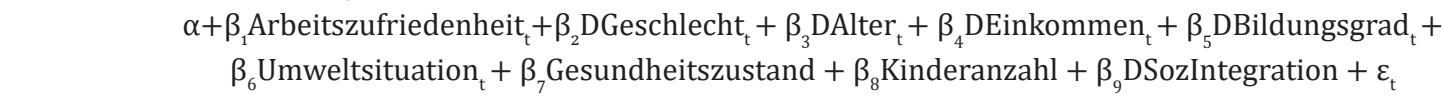 } \\
\hline Prädiktor & Nichtstand. Reg. Koeffiz. & $\mathrm{T}$ & $\mathrm{p}$ \\
\hline Konstante &, 554 &, 833 &, 405 \\
\hline Arbeitszufriedenheit & ,371 & 8,689 & $<, 001$ \\
\hline Geschlecht & ,232 & 1,482 & 139 \\
\hline Alter & ,259 & 1,496 & 135 \\
\hline Einkommen & ,685 & 2,412 &, 016 \\
\hline Bildungsgrad & ,166 &, 573 & ,567 \\
\hline Umweltsituation & ,159 & 3,053 &, 002 \\
\hline Gesundheitszustand & ,220 & 4,618 & $<, 001$ \\
\hline Anzahl der Kinder &,- 153 & $-1,669$ & 096 \\
\hline Soziale Integration & ,399 & ,726 & ,469 \\
\hline
\end{tabular}

Anmerkung: Vollständige Ergebnisauflistung siehe Anhang: Regression 2

Laut ANOVA (F-Test $=148,8, \mathrm{p}<, 001)$ wird die Nullhypothese, das Modell habe keine Erklärungskraft, deutlich verworfen. Dementsprechend zeigen sich Konstante und unabhängige Variable jeweils als hoch signifikant. Eine um eine Einheit höhere Selbstangabe der Arbeitszufriedenheit geht laut Schätzung mit einer Erhöhung der Lebenszufriedenheit um etwa 0,44 Skalen-Einheiten einher.

Zur Prüfung der Frage, ob die Arbeitszufriedenheit für die gesamte Lebenszufriedenheit eine zentrale Bedeutung einnimmt, wird die Abhängigkeit der allgemeinen Lebenszufriedenheit von einer Reihe anderer Variablen untersucht, denen in der Forschung hohe Erklärungskraft zugesprochen wird. ${ }^{10}$ Mittels multipler Regression werden die Einflussstärken des berichteten Gesundheitszustands, die Einschätzung der Umweltsituation, das Ausmaß der selbstberichteten sozialen Integration sowie die Einkommenssituation ${ }^{11}$ untersucht und für soziodemografische Variablen Geschlecht, Alter, Bildungsgrad und Anzahl der Kinder kontrolliert.

Das Modell wird wie folgt geschätzt:

10 Vgl. OECD-Better-Life-Index, Quality of Life Index, Eurostat

11 Variable gebildet aus beruflicher Stellung und Bildungsstand.
Lebenszufriedenheit $=$ $\alpha+\beta_{1}$ Arbeitszufriedenheit $+\beta_{2}$ DGeschlecht $t_{t}$ $+\beta_{3}$ DAlter $_{t}+\beta_{4}$ DEinkommen $_{t}+\beta_{5}$ DBildungsgrad $_{t}$ $+\beta_{6}$ Umweltsituation $_{t}+\beta_{7}$ Gesundheitszustand + $\beta_{8}$ Kinderanzahl $+\beta_{9}$ DSozIntegration $+\varepsilon_{t}$

Die ANOVA zeigt die Signifikanz des Modells. Rund $37 \%$ der Varianz der Lebenszufriedenheit (korrigiertes $\mathrm{R}^{2}$ : 0,372) wird durch die im Modell enthaltenen Variablen erklärt, $63 \%$ bleiben unbekannt. ${ }^{12}$ Ein Anteil der durch das Modell erklärten Varianz von 37 \% liegt im Bereich der Ergebnisse vergleichbarer Studien. Boarini et.al berichten $\mathrm{R}^{2}$-Werte von o,35 für zwei Wellen des Gallup World Poll für 34 OECD-Länder unter Einbezug einer großen Zahl erklärender Variablen für die allgemeine Lebenszufriedenheit (Boarini,Comola, Smith, Manchin, De Keulenaer 2012). Fleche, Smith und Sorsa (2011) kamen in vergleichenden Länderstudien auf der Grundlage von drei Wellen der World Value Surveys für die OECD-Länder auf einen durchschnittlichen $\mathrm{R}^{2}$-Wert von 0,22 (Fleche, Smith, Sorsa 2011)

Interessant ist, dass sich die bekundete Arbeitszufriedenheit (innerhalb des Modells) nicht nur als wich-

12 Der signifikante F-Wert $(26,245$, p-Wert: <,oo1 $)$ zeigt jedoch deutlich, dass die Nullhypothese, das Modell habe keine Erklärungskraft, nicht haltbar und zu verwerfen ist. 


\begin{tabular}{|c|c|c|c|}
\hline & \multicolumn{3}{|c|}{ Faktoren } \\
\hline & 1 & 2 & 3 \\
\hline Es geht mir nicht gut, aber andere haben gar keine Arbeit. &,- 724 & & \\
\hline Ich komme zurecht. Es kann so bleiben, wie es ist. &, 590 & &, 539 \\
\hline Mein Arbeitsplatz ist sicher. & ,586 & & \\
\hline Berufliche Herausforderungen spornen mich an. & & 806 & \\
\hline Mir ist wichtig, dass mir meine Arbeit Freude macht. & & ,797 & \\
\hline Ich empfinde meine Arbeit als sinnvoll. & & ,560 & \\
\hline Beruflich läuft es gut, und ich werde noch einiges erreichen. & &, 557 & \\
\hline Ich habe mich mit meiner beruflichen Situation abgefunden. & & & ,774 \\
\hline Am wichtigsten ist es, gesund in die Pension zu kommen. & & &, 622 \\
\hline Die hohe Arbeitslosigkeit macht mir Sorgen. & & & \\
\hline
\end{tabular}

tiger, sondern nach dem Einkommen zweitwichtigster Prädiktor der Lebenszufriedenheit erweist $(\beta=, 371, \mathrm{p}<$ ,oo1). Mit deutlichem Abstand folgen der Gesundheitszustand $(\beta=, 220, p<, 001)$ und die wahrgenommene Umweltsituation $(\beta=, 159, \mathrm{p}=, 002){ }^{13}$ Die Prädiktoren Arbeitszufriedenheit, Einkommen, Umweltqualität und Gesundheitszustand können auf dem 5 \%-Signifikanzniveau abgesichert werden. Die Variablen Geschlecht, Alter, Bildung, Anzahl der Kinder und soziale Integration erweisen sich in dem vorliegenden Modell hingegen nicht als signifikant. Zur Kontrolle wurden die Variablen Arbeitszufriedenheit, Einkommen, Umweltqualität und Gesundheitszustand schrittweise auf die Lebenszufriedenheit regrediert. Im Ergebnis zeigt eine Varianzerklärung der Lebenszufriedenheit von $37 \%$.

\subsection{Führen objektiv gute Arbeitsverhältnisse zu hoher Arbeitszufriedenheit?}

Kommt angesichts dieses hohen Stellenwerts der umfassenden Arbeitszufriedenheit für die gesamte Lebenszufriedenheit aber auch zum Ausdruck, dass es den Menschen in ihrer Arbeit auch tatsächlich gut geht? Lässt sich von hohen Werten bei der Frage nach der umfassenden Arbeitszufriedenheit umstandslos auch auf eine gute Arbeitssituation schließen? Unter Bezugnahme etwa auf Bruggemanns Modell der Arbeitszufriedenheit wäre ja denkbar, dass sich Beschäftigte mit ihrer Situation abgefunden und arrangiert haben und ihre Ansprüche und Erwartungen an ihr konkretes Arbeitsleben reduziert haben. Diesem Zufriedenheitsmodell folgend, bei dem Zufriedenheit aus einer Über-

13 Test auf Multikollinearität negativ. einstimmung von Ansprüchen sowie Erwartungen und der tatsächlichen Realität resultiert, würde eine Reduktion von Ansprüchen und Erwartungen auch bei geringerer Qualität des Arbeitslebens zu hoher Zufriedenheit führen.

Zur Überprüfung der Frage, ob es Beschäftigten, die hohe Arbeitszufriedenheit bekunden, auch tatsächlich gut geht - sie also motiviert und mit Freude einer sinnerfüllten Beschäftigung nachgehen -, ist es daher nötig, mehr über die tatsächliche Befindlichkeit der Beschäftigten zu erfahren. Im Rahmen der Stichprobe wurden die Erwerbstätigen mit elf Aussagen zu ihrem aktuellen Arbeitsleben konfrontiert. Die Aussagen umspannten sowohl affektive als auch kognitive Komponenten der Zufriedenheit und beziehen sich auf Gedanken, Gefühle und Einstellungen hinsichtlich Sinnerfüllung, Motivation, Erwartungen und Ziele an das Arbeitsleben. Die Befragten bewerteten wiederum auf einer Skala von o bis 10, inwiefern die beschriebenen Aussagen auf sie persönlich zutreffen. Mittels einer Faktorenanalyse wurden die Aussagen zur Arbeitssituation zu einer kleineren Zahl von unabhängigen Einflussgrößen reduziert, wobei drei Faktoren extrahiert wurden. Inhaltlich werden dabei jene Variablen, die stark untereinander korrelieren, zu einem Faktor generiert. Tabelle 4 zeigt anhand der Komponentenmatrix der Faktoranalyse, wie stark die Aussagen zum Arbeitsleben auf die drei extrahierten Faktoren laden. Der erste Faktor erklärt rund $30 \%$ der Gesamtvarianz, der zweite Faktor rund $16 \%$ und der dritte Faktor rund $11 \%{ }^{14}$

14 Extraktionsmethode: Hauptkomponentenanalyse; Rotationsmethode: Varimax mit Kaiser-Normalisierung; KMO-Test: ,775; Signifikanz Bartlett: $\mathrm{p}<, 001$. 
Tabelle 5 Korrelationskoeffizienten unterschiedlicher Arbeitssituationen und der Arbeitszufriedenheit sowie Lebenszufriedenheit

\begin{tabular}{|c|c|c|c|c|}
\hline \multirow[b]{2}{*}{ Faktor } & \multicolumn{2}{|c|}{ Arbeitszufriedenheit } & \multicolumn{2}{|c|}{ Lebenszufriedenheit } \\
\hline & $\mathrm{r}$ & $\mathrm{p}$ & $\mathrm{r}$ & $\mathrm{p}$ \\
\hline Stabiles, unmotiviertes Arbeitsleben &, 531 & $\mathrm{p}<, 001$ &, 430 & $\mathrm{p}<, 001$ \\
\hline Positives, motiviertes Arbeitsleben &, 463 & $\mathrm{p}<, 001$ & ,240 & $\mathrm{p}<, 001$ \\
\hline schlechtes, demotiviertes Arbeitsleben & , 164 & $\mathrm{p}<, 001$ &, 112 & $p<, 001$ \\
\hline
\end{tabular}

Der inhaltliche Zusammenhang des ersten Faktors bringt ein emotional distanziertes und nüchternes Verhältnis zur beruflichen Tätigkeit zum Ausdruck. Dieser Faktor lässt sich als „stabiles, aber unmotiviertes Arbeitsleben" beschreiben: Variablen, die Motivation oder Sinnerfüllung ausdrücken, laden nicht auf diesen Faktor. Der Arbeitsplatz bietet zwar wenig Herausforderungen, wird aber als sicher bewertet. Auffällig ist, dass die Variablen „Derzeit läuft es schlecht [...]“ und „Es geht mir nicht gut $[\ldots]$ “ besonders negativ auf diesen Faktor laden. Faktor 2 bringt Motivation und Arbeitsfreude zum Ausdruck und lässt sich kurz als „positives, motiviertes Arbeitsleben“ beschreiben. Der Faktor umschreibt eine motivierte („[...] Herausforderungen spornen mich an“) und zuversichtliche („[...] werde noch einiges erreichen") Beschreibung der Arbeitssituation. Die Arbeit ist sinnerfüllt und bereitet Freude. Faktor 3 lässt sich kurz als „resignatives Arbeitsleben“ beschreiben. Personen mit hohen Werten bei Faktor 3 haben sich mit ihrer Situation arrangiert („[...] habe mich abgefunden“). Die Erwartungen beziehen sich auf die Zeit jenseits des Arbeitslebens („Am wichtigsten ist es, gesund in die Pension zu kommen."). Auch auf diesen Faktor lädt die Variable „Ich komme zurecht.

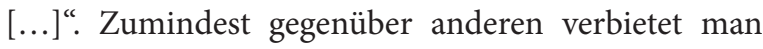
sich, die mäßige Arbeitssituation zu beklagen.

Bewerten Personen mit hohen Werten beim Faktor „resignatives Arbeitsleben“ ihre umfassende Arbeitszufriedenheit nun als gering? Und umgekehrt, bewerten Personen mit hohen Werten des Faktors „positives, motivierendes Arbeitsleben“ ihre Arbeitszufriedenheit als hoch? Zu erwarten wäre, dass eine mäßige und resignative Arbeitssituation stark mit einer geringen subjektiven Arbeitszufriedenheit korreliert, eine progressive, starke Arbeitssituation dagegen mit hoher Arbeitszufriedenheit einhergeht. Zur Prüfung dieser Frage wurden für die drei in der Faktorenanalyse extrahierten Faktoren, mithilfe der jeweiligen Faktorladungen, die jeder Person entsprechenden Faktorwerte berechnet und als eigene Variable abgespeichert. Anschließend wurden diese Werte mit den entsprechenden Angaben zur subjektiven Arbeitszufriedenheit korreliert. Tabelle
5 zeigt die Korrelationskoeffizienten für umfassende Arbeitszufriedenheit als auch für umfassende Lebenszufriedenheit. Alle Korrelationskoeffizienten weisen ein positives Vorzeichen auf. Dies ist irritierend, denn es bedeutet, dass jeder Faktor positiv mit subjektiver Arbeitszufriedenheit (wenn auch in unterschiedlichem Ausmaß) korreliert.

Ein stabiles, aber unmotiviertes Arbeitsleben (Faktor 1) korreliert am stärksten mit Arbeitszufriedenheit $(\mathrm{r}=, 531 ; \mathrm{p}<, 001)$ und damit stärker als ein positives, motiviertes Arbeitsleben $(r=, 463$; $p<, 001)$. Offenbar ist der Zusammenhang zwischen Zufriedenheit und einer als wenig euphorisch, aber als sicher und stabil empfundenen Arbeitssituation stärker, auch wenn die Arbeitssituation nicht als motivierend oder sinnvoll empfunden wird. Zwei Variablen laden deutlich negativ auf diesen Faktor und zwar jene, die eine schlechte Bewertung der Arbeitssituation ausdrücken. Personen mit hohen Werten bei Faktor 1 (stabiles, aber unmotiviertes Arbeitsleben) stimmen Aussagen, die zum Ausdruck bringen, dass es schlecht läuft, wenig zu. Personen, die bei diesem Faktor hohe Werte aufweisen, wollen trotz mäßiger Motivation und geringen Ansprüchen und Perspektiven offenbar keinesfalls jammern. Offenbar verbietet sich eine schlechte Bewertung der eigenen Arbeitssituation, zumindest wird es als unangebracht empfunden, das in einer Befragung zu vermitteln. Ein herausforderndes, freud- und sinnvolles Arbeitsleben (Faktor 2) korreliert ebenfalls mit einer hohen Arbeitszufriedenheit, erstaunlicherweise aber geringer als die zuvor beschriebene nüchterne und weniger motivierte Arbeitssituation. Das deutet darauf hin, dass Motivation, Freude und Herausforderungen im Arbeitsleben zwar wichtige Korrelate der Arbeitszufriedenheit abgeben, aber keineswegs wichtigere als Sicherheit und Stabilität der Arbeitssituation. Das bestätigt auch der Blick auf die Bewertung der gesamten Lebenszufriedenheit. Ein stabiles, aber wenig motivierendes Arbeitsverhältnis korreliert stärker mit der gesamten Lebenszufriedenheit $(r=, 460 ; p<, 001)$ als eine motivierende und sinnerfüllte Arbeitssituation $(\mathrm{r}=, 240 ; \mathrm{p}<, 001)$. 


\begin{tabular}{|c|c|c|c|}
\hline \multicolumn{4}{|c|}{ Modellzusammenfassung } \\
\hline $\mathrm{F}$ & $\mathrm{p}$ & Korrigiertes $\mathrm{R}^{2}$ & N \\
\hline 72,434 & $<, 001$ &, 594 & 342 \\
\hline \multicolumn{4}{|c|}{$\alpha+\beta_{1}$ Faktor $1_{t}+\beta_{2}$ Faktor $2_{t}+\beta_{3}$ Faktor $3_{t}+\beta_{4}$ DGeschlecht $_{t}+\beta_{5}$ Alter $_{t}+\beta_{6}$ DEinkommen $_{t}+\beta_{7}$ DBildungsgrad $+\varepsilon_{t}$} \\
\hline Prädiktor & Nichtstand. Reg. Koeffiz. & $\mathrm{T}$ & $\mathrm{p}$ \\
\hline Konstante & 7,491 & 55,208 & $\mathrm{p}<, 001$ \\
\hline Faktor 1 & 1,210 & 17,285 & $\mathrm{p}<, 001$ \\
\hline Faktor 2 & 917 & 13,035 & $\mathrm{p}<, 001$ \\
\hline Faktor 3 & ,421 & 5,408 & $\mathrm{p}<, 001$ \\
\hline Geschlecht &,- 131 &,- 926 & ,355 \\
\hline Alter &, 039 & ,251 & ,802 \\
\hline Einkommen &,- 453 & $-1,817$ & , 070 \\
\hline Bildung & ,312 & 1,227 & ,221 \\
\hline
\end{tabular}

Anmerkung: Vollständige Ergebnisauflistung siehe Anhang: Regression 3

Auch für die im Arbeitsleben Resignierten (Faktor 3) lässt sich ein positiver, wenn auch schwacher Zusammenhang sowohl mit hoher Arbeitszufriedenheit als auch mit hoher Lebenszufriedenheit feststellen. Folgt man der Hypothese, dass sich schlecht bewertete Arbeitsbedingungen in einem entsprechenden Zufriedenheitsurteil abbilden, wäre ein negatives Vorzeichen $\mathrm{zu}$ erwarten gewesen. Tatsächlich ist eine resignierte Arbeitseinstellung mit hoher allgemeiner Arbeitszufriedenheit vereinbar.

Wie hoch nun ist der Anteil der Beschäftigten, auf den dieses Muster zutrifft? Die Kreuzung (Kreuztabelle siehe Anhang) von Faktor 3 und gesamter Arbeitszufriedenheit zeigt, dass $29 \%$ der Personen, die eine sehr hohe Arbeitszufriedenheit bekunden, ${ }^{15}$ stark auf Faktor 3 laden, und $31 \%$ dieser Personengruppe leicht auf Faktor 3 laden. Insgesamt zeigt damit eine deutliche Mehrheit der Personen mit einer hohen Arbeitszufriedenheit eine leichte bis starke positive Ladung auf den Faktor der resignierten Arbeitseinstellung. Das sind $15,9 \%{ }^{16}$ aller Befragten.

Zur Prüfung der Ergebnisse der Korrelationsanalyse wurde eine Regression gerechnet, bei der die drei Faktoren der Faktorenanalyse in ein Modell der Arbeitszufriedenheit eingehen und um sozioökonomi-

15 Also einen Wert von 9 oder 10 auf der Arbeitszufriedenheitsskala von o bis 10 angaben.

16 Gültige Prozent sche Aspekte (Geschlecht, Alter, Bildung und Einkommen $\left.^{17}\right)$ kontrolliert werden.

Arbeitszufriedenheit $_{t}=$

$\alpha+\beta_{1}$ Faktor $1_{t}+\beta_{2}$ Faktor $2_{t}+\beta_{3}$ Faktor $3_{t}+\beta_{4}$ DGeschlecht $t_{t}$

$+\beta_{5}$ Alter $_{t}+\beta_{6}$ DEinkommen $_{t}+\beta_{7}$ DMatura $+\varepsilon_{t}$

Mit einer durch das Modell erklärten Varianz der abhängigen Variable von beinahe 60 \% (korrigiertes $\mathrm{R}^{2}$ ) kann von einer sehr guten Anpassungsgüte des Modells gesprochen werden. Alle Modellanforderungen sind getestet und erfüllt.

Laut ANOVA (F-Test $=72,434, \mathrm{p}<, 001)$ ist die Nullhypothese, das Modell habe keine Erklärungskraft, nicht haltbar. So zeigen sich auch insbesondere die drei Faktoren als deutlich signifikante Einflussvariablen. Wie bereits die Korrelationen der Faktoren mit der Arbeitszufriedenheit zeigten, wirkt sich insbesondere Faktor 1 stark auf die Arbeitszufriedenheit aus. Auch wird das Ergebnis eines für alle Faktoren positiven Vorzeichens bestätigt. Von den sozioökonomischen Kontrollvariablen zeigt sich auf einem fünfprozentigen Signifikanzniveau keine als signifikant. Lediglich die Einkommensdummy liegt knapp über diesem Signifikanzniveau. Eine Regression ohne sozioökonomische Variablen zeigt, dass über $53 \%$ der Varianz der Arbeitszufriedenheit durch die Faktoren 1,2 und 3 erklärt werden kann.

17 Als Proxy für Einkommen wurde eine Variable aus beruflicher Stellung und höchster Ausbildung konstruiert. 
6.3 Wie können diese Ergebnisse interpretiert werden?

Stabilität und Sicherheit des Arbeitsplatzes sind die wichtigsten Determinanten hoher Arbeitszufriedenheit. Für einen sehr großen Teil der Beschäftigten resultiert hohe Arbeitszufriedenheit aus materiellen und arbeitsplatzbezogenen Sicherheitsbedürfnissen, während Entwicklungsperspektiven und intrinsische Motivation eine wichtige, aber eher nachrangige Rolle spielen. Diese Gruppe hat sich an die Verhältnisse angepasst und schafft es offenbar, die Balance zwischen Anforderungen und Arbeitseinsatz stabil zu halten. Interessanterweise stimmen Personen mit hohen Werten beim Faktor 1 Aussagen, die zum Ausdruck bringen, dass es schlecht läuft, gar nicht zu. Offenbar verbietet sich eine schlechte Bewertung der eigenen Arbeitssituation, zumindest wird es als unangebracht empfunden, das in einer Befragung $\mathrm{zu}$ vermitteln. Motivation, Freude und ein herausforderndes Arbeitsleben (Faktor 2) ist eine weitere wichtige Determinante der Arbeitszufriedenheit, aber keineswegs wichtiger als Sicherheit und Stabilität der Arbeitssituation. Grundbedürfnisse des Arbeitslebens, wie ausreichende Gestaltungs- und Handlungsmöglichkeiten, sind vorhanden. Die Balance zwischen Arbeitseinsatz und Gratifikationen inklusive Entwicklungsperspektiven ist gegeben.

Auch eine resignierte Arbeitseinstellung trägt zur Erklärung der Arbeitszufriedenheit bei. Das kontraintuitivste Ergebnis der Untersuchung ist wohl, dass Personen, deren Arbeitseinstellung als demotiviert bezeichnet werden kann und die keine Versuche unternehmen, an ihrem Zustand etwas zu verändern, ihre gesamte Arbeitszufriedenheit nicht überwiegend negativ, sondern positiv einschätzen. Die Werte der Kreuztabelle (siehe Anhang) zeigen auch, dass es sich bei dieser Gruppe von Beschäftigten keineswegs um eine kleine Randgruppe handelt. Offenbar wollen Beschäftigte, denen es im Arbeitsleben nicht gut geht, Unzufriedenheit vermeiden. Unzufriedenheit wird als Störung des Gleichgewichtes empfunden und somit als ein Spannungszustand, den es zu beseitigen gilt. Dissonanzen erzeugen einen Druck zu ihrer Reduktion (Festinger 1957: 3). Wird Unzufriedenheit mit der Arbeitssituation als Diskrepanz zwischen einem Anspruch (Soll) und einer Einschätzung der Realität (Ist) gesehen, dann findet ein Ausgleich zwischen Sollund Ist-Zustand offenbar über eine Herabregulierung der Erwartungen, Ansprüche und Ziele statt und wirkt somit gewissermaßen als gesunderhaltende Vermeidungsstrategie kognitiver Dissonanz. Diese Diskrepanz zwischen „Soll“ und „Ist“ ist mitunter nicht nur schwer zu ertragen, und selbst wenn zu ertragen, „nicht etwas, das in der Interviewsituation ohne weiteres einem fremden Menschen mitgeteilt wird“ (Hofinger 2007: 48). Die Adaption der Erwartungen und Ansprüche an eine mäßig oder gar schlecht bewertete Arbeitsrealität ermöglicht somit ein verbessertes Zufriedenheitsurteil.

Die hier festgestellte, formal positive Ausprägung von Arbeitszufriedenheit trotz mäßiger Arbeitsbedingungen stimmt durchaus mit anderen jüngeren Ergebnissen der Messung von Arbeitszufriedenheit überein. So resümieren etwa Fischer und Belschak als Ergebnis ihrer Untersuchungen zur Arbeitszufriedenheit: „Selten wurde eine überzogene Klagsamkeit, dagegen häufiger eine typische ,kognitive Selbstheilung' festgestellt" (Fischer/Belschak 2006: 81).

\section{Zusammenfassende abschließende Überlegungen}

- Die vorliegende Analyse einer Befragung von Beschäftigten zu ihrer Arbeitszufriedenheit hat gezeigt, dass hinter formal gleicher und hoher Arbeitszufriedenheit diverse innerpsychische Prozesse stattfinden, bei denen Informationen aus dem Arbeitsleben unterschiedlich wahrgenommen, verarbeitet und bewertet werden.

- Hohe und höchste Arbeitszufriedenheit kann sowohl auf ein freudvolles, herausforderndes und sinnerfülltes Arbeitsleben als auch auf eine Haltung zurückgeführt werden, bei der vor allem ökonomische und soziale Sicherheitsbedürfnisse vorherrschend sind und man sich mit den Verhältnissen abgefunden hat. Auch Auf- und Abwärtsvergleiche mit Vergleichsgruppen können hier angesichts eines wachsenden gesellschaftlichen Unsicherheitsgefühls zum Zufriedenheitsurteil beitragen. Selbst ein demotiviertes und perspektivenloses Arbeitsleben kann zu höchster Zufriedenheit führen, zu resignativer Zufriedenheit, bei der Beschäftigte nur noch darauf fokussiert sind, ihr Arbeitsleben möglichst unversehrt zu Ende zu führen.

- Wie andere „Bereichszufriedenheiten“ der Lebensqualität (Diener 1999) kann eine reflektierte allgemeine Arbeitszufriedenheit auf Basis eines SollIst-Vergleichs modelliert werden. Der dahinterliegende Prozess der Urteilsbildung muss aber komplex gedacht werden, denn das Zufriedenheitsurteil ist nicht einfach als Saldo zwischen einem einmal definierten Anspruch 
und einer wahrgenommenen Arbeitswirklichkeit zu deuten. Dagegen fließen eigene, wandelbare, individuelle Ansprüche, Vergleichshorizonte und Bezugssysteme in die Bewertung der gesamten Arbeitssituation als intervenierende Variable genauso mit ein wie vielfältige, von der eigenen Arbeitssituation unabhängige gesellschaftliche Wertmaßstäbe eines guten Lebens als Referenzmaßstab. Eine hohe Arbeitszufriedenheit ist in Arbeitsgesellschaften gerade in Zeiten hoher allgemeiner Verunsicherung in hohem Ausmaß sozial erwünscht. Angesichts der hohen Bedeutung von Erwerbsarbeit werden, wie die Ergebnisse dieser Untersuchung zeigen, selbst die kritikwürdigsten Verhältnisse von den Erwerbstätigen nicht gänzlich abgewertet.

- Der ausschließliche Blick auf den Wert der gemessenen umfassenden Arbeitszufriedenheit sagt selbst dann, wenn er als Quotient aus Anspruchsniveau und Arbeitsbedingungen interpretiert wird, noch nichts über die unterschiedlichen Aspekte der Arbeitszufriedenheit und deren tatsächliche Größen aus. Arbeitszufriedenheit als Folge schlechter Arbeitsbedingungen, aber niedriger Ansprüche, kann denselben Wert erreichen wie beste Arbeitsbedingungen bei höchsten Ansprüchen. Nur umfassendere Messinstrumente, die die Vielfalt arbeitsrelevanter Einflussvariablen differenzierter berücksichtigen, sind daher geeignet, Arbeitszufriedenheit adäquat abzubilden.

- Jedenfalls kann von hohen Werten bei der singulären Frage nach der umfassenden Arbeitszufriedenheit nicht einfach auf eine objektiv gute Arbeitssituation oder eine durchgängig gute Bewertung des Arbeitslebens geschlossen werden, zumindest dann nicht, wenn zur Arbeitsqualität nicht nur äußere, objektvierbare Merkmale der Arbeit, sondern auch die darauf bezogenen Kognitionen gezählt werden. Die Motive, die $\mathrm{zu}$ hoher Arbeitszufriedenheit führen, sind plural geworden.

- Methodisch liefern die Ergebnisse der vorliegenden Untersuchung ausreichende Hinweise (das kann zumindest für die Messung der Arbeitszufriedenheit gelten), dass Single-Item-Befragungen zur Zufriedenheit, wie das in diversen Wohlfahrtsmaßen geschieht, als Indikator für die gesamte Arbeitszufriedenheit untauglich sind, denn sie unterschätzen die Komplexität des Konstrukts Arbeitszufriedenheit fundamental. Darüber hinaus bieten sie Gelegenheit zu Fehldeutungen wie eben jene, dass hohe Zufriedenheitswerte auf eine gute Bewertung der Arbeitsbedingungen schließen lassen und damit auch zu interessenpolitisch absichtsvollen Interpretationen.
- Die permanent hohen Werte der Arbeitszufriedenheit trotz unsicherer Rahmenbedingungen sind wesentlich als eine Folge von Anpassungsreaktionen zu verstehen, mit denen Menschen versuchen, eine schlechte Bewertung ihrer Zufriedenheit zu vermeiden. Bei einem Arrangement, also einer Anpassung an die gegebenen äußeren Bedingungen, verändern sich auch die mit dem Job verbundenen Anschauungen und Emotionen, was bei der Interpretation des Zufriedenheitsurteils berücksichtigt werden muss.

- Die von der Postmoderne noch vor wenigen Jahrzehnten popularisierte These eines allgemeinen Wertewandels weg von materiellen Sicherungs- und Statusbedürfnissen hin zu Selbstverwirklichungsbedürfnissen kann als überholt gelten. Die Bedeutung der eigenen Arbeit für die subjektive Lebensqualität ist nicht nur nach wie vor hoch, sondern zu traditionellen Arbeitswerten treten neue hinzu bzw. gewinnen alte in Phasen gesellschaftlicher Verunsicherung neuen Auftrieb. Dabei spielen soziale und ökonomische Sicherungsbedürfnisse nicht nur unterhalb einer klar zu definierender Sozialstrukturgrenze eine große Rolle. 


\section{Anhang}

\subsection{Kreuztabellen}

\begin{tabular}{|c|c|c|c|c|c|c|c|}
\hline \multicolumn{8}{|c|}{ Arbeitszufriedenheit * Faktor 1 Kreuztabelle } \\
\hline & & & Faktor 1 (klass & jert in 4 Klassen) & & & \\
\hline & & & $\begin{array}{l}\text { stark negativ } \\
(<=-1)\end{array}$ & $\begin{array}{l}\text { leicht negativ } \\
(<=0)\end{array}$ & $\begin{array}{l}\text { leicht positiv } \\
(<=1)\end{array}$ & $\begin{array}{l}\text { stark positiv } \\
(<=2,5)\end{array}$ & Gesamt \\
\hline \multirow[t]{6}{*}{ Arbeitszufriedenheit } & gering $(0-5)$ & Anzahl & 35 & 32 & 10 & 0 & 77 \\
\hline & & $\%$ innerhalb Kat. & $45,5 \%$ & $41,6 \%$ & $13,0 \%$ & $0,0 \%$ & $100,0 \%$ \\
\hline & mittel (6-8) & Anzahl & 31 & 98 & 93 & 36 & 258 \\
\hline & & $\%$ innerhalb Kat. & $12,0 \%$ & $38,0 \%$ & $36,0 \%$ & $14,0 \%$ & $100,0 \%$ \\
\hline & hoch $(9-10)$ & Anzahl & 7 & 25 & 65 & 48 & 145 \\
\hline & & $\%$ innerhalb Kat. & $4,8 \%$ & $17,2 \%$ & $44,8 \%$ & $33,1 \%$ & $100,0 \%$ \\
\hline \multirow[t]{2}{*}{ Gesamt } & & Anzahl & 73 & 155 & 168 & 84 & 480 \\
\hline & & $\%$ innerhalb Kat. & $15,2 \%$ & $32,3 \%$ & $35,0 \%$ & $17,5 \%$ & $100,0 \%$ \\
\hline \multicolumn{8}{|c|}{ Arbeitszufriedenheit * Faktor 2 Kreuztabelle } \\
\hline \multirow{6}{*}{ Arbeitszufriedenheit } & & & Faktor 2 (klass & iert 4 Klassen) & & & \\
\hline & & & $\begin{array}{l}\text { stark negativ } \\
(<=-1)\end{array}$ & $\begin{array}{l}\text { leicht negativ } \\
(<=0)\end{array}$ & $\begin{array}{l}\text { leicht positiv } \\
(<=1)\end{array}$ & $\begin{array}{l}\text { stark positiv } \\
(<=2,5)\end{array}$ & Gesamt \\
\hline & gering $(0-5)$ & Anzahl & 30 & 25 & 15 & 7 & 77 \\
\hline & & $\%$ innerhalb Kat. & $39,0 \%$ & $32,5 \%$ & $19,5 \%$ & $9,1 \%$ & $100,0 \%$ \\
\hline & mittel (6-8) & Anzahl & 40 & 90 & 95 & 33 & 258 \\
\hline & & $\%$ innerhalb Kat. & $15,5 \%$ & $34,9 \%$ & $36,8 \%$ & $12,8 \%$ & $100,0 \%$ \\
\hline \multirow{4}{*}{ Gesamt } & hoch $(9-10)$ & Anzahl & 4 & 20 & 83 & 38 & 145 \\
\hline & & $\%$ innerhalb Kat. & $2,8 \%$ & $13,8 \%$ & $57,2 \%$ & $26,2 \%$ & $100,0 \%$ \\
\hline & & Anzahl & 74 & 135 & 193 & 78 & 480 \\
\hline & & $\%$ innerhalb Kat. & $15,4 \%$ & $28,1 \%$ & $40,2 \%$ & $16,3 \%$ & $100,0 \%$ \\
\hline \multicolumn{8}{|c|}{ Arbeitszufriedenheit * Faktor 3 Kreuztabelle } \\
\hline \multirow{7}{*}{ Arbeitszufriedenheit } & & & Faktor 3 (klass & iert 4 Klassen) & & & \\
\hline & & & $\begin{array}{l}\text { stark negativ } \\
(<=-1)\end{array}$ & $\begin{array}{l}\text { leicht negativ } \\
(<=0)\end{array}$ & $\begin{array}{l}\text { leicht positiv } \\
(<=1)\end{array}$ & $\begin{array}{l}\text { stark positiv } \\
(<=2,5)\end{array}$ & Gesamt \\
\hline & gering $(0-5)$ & Anzahl & 18 & 30 & 21 & 8 & 77 \\
\hline & & $\%$ innerhalb f10 & $23,4 \%$ & $39,0 \%$ & $27,3 \%$ & $10,4 \%$ & $100,0 \%$ \\
\hline & mittel (6-8) & Anzahl & 37 & 85 & 102 & 34 & 258 \\
\hline & & \% innerhalb f10 & $14,3 \%$ & $32,9 \%$ & $39,5 \%$ & $13,2 \%$ & $100,0 \%$ \\
\hline & hoch (9-10) & Anzahl & 20 & 38 & 45 & 42 & 145 \\
\hline \multirow{3}{*}{ Gesamt } & & $\%$ innerhalb f10 & $13,8 \%$ & $26,2 \%$ & $31,0 \%$ & $29,0 \%$ & $100,0 \%$ \\
\hline & & Anzahl & 75 & 153 & 168 & 84 & 480 \\
\hline & & $\%$ innerhalb f10 & $15,6 \%$ & $31,9 \%$ & $35,0 \%$ & $17,5 \%$ & $100,0 \%$ \\
\hline
\end{tabular}




\subsection{Regression 1}

\begin{tabular}{llll}
\hline \multicolumn{3}{c}{ Aufgenommene/Entfernte Variablen $^{\text {a }}$} & \\
\hline Modell & Aufgenommene Variablen & Entfernte Variablen & Methode \\
1 & Wie zufrieden sind Sie insgesamt mit Ihrer beruflichen Tätigkeit? & & \\
& Ziffer $0=$ überhaupt nicht zufrieden, $10=$ sehr zufrieden.b & Einschluss \\
\hline
\end{tabular}

a. Abhängige Variable: Ihrer Lebensqualität

b. Alle gewünschten Variablen wurden eingegeben.

\begin{tabular}{lccccc}
\hline \multicolumn{5}{c}{ Modellzusammenfassung $^{b}$} \\
\hline Modell & $\mathrm{R}$ & R-Quadrat & Korrigiertes R-Quadrat & Standardfehler des Schätzers & Durbin-Watson-Statistik \\
1 &, $463^{\mathrm{a}}$ &, 215 &, 213 & 1,714 & 1,832 \\
\hline
\end{tabular}

a. Einflussvariablen : (Konstante), Wie zufrieden sind Sie insgesamt mit Ihrer beruflichen Tätigkeit? Ziffer o = überhaupt nicht zufrieden, $10=$ sehr zufrieden.

b. Abhängige Variable: Ihrer Lebensqualität

\begin{tabular}{lllllll}
\hline & & \multicolumn{5}{c}{ ANOVA $^{\text {a }}$} \\
\hline Modell & & Quadratsumme & $\mathrm{df}$ & Mittel der Quadrate & $\mathrm{F}$ & Sig. \\
1 & Regression & 437,332 & 1 & 437,332 & 148,837 &, $000 \mathrm{~b}$ \\
& Nicht standardisierte Residuen & 1601,385 & 545 & 2,938 & \\
& Gesamt & 2038,717 & 546 & & \\
\hline
\end{tabular}

a. Abhängige Variable: Ihrer Lebensqualität

b. Einflussvariablen : (Konstante), Wie zufrieden sind Sie insgesamt mit Ihrer beruflichen Tätigkeit? Ziffer o = überhaupt nicht zufrieden, $10=$ sehr zufrieden .

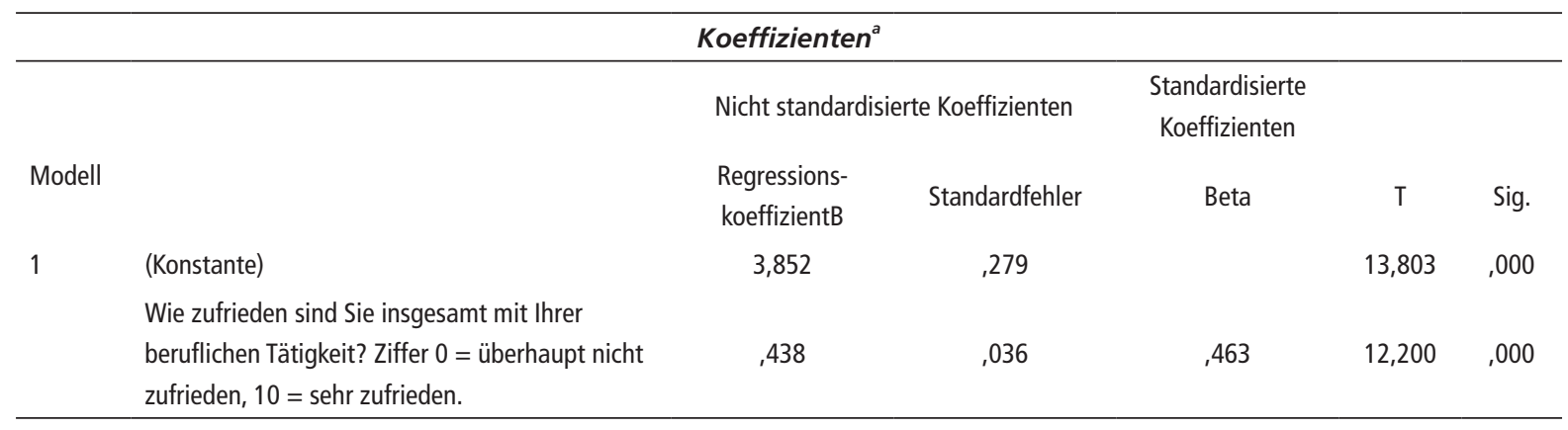

a. Abhängige Variable: Ihrer Lebensqualität

\begin{tabular}{|c|c|c|c|c|c|}
\hline \multicolumn{6}{|c|}{ Residuenstatistik ${ }^{a}$} \\
\hline & Minimum & Maximum & Mittelwert & Standardabweichung & $\mathrm{N}$ \\
\hline Nicht standardisierter vorhergesagter Wert & 3,85 & 8,23 & 7,14 &, 895 & 547 \\
\hline Nicht standardisierte Residuen & $-5,920$ & 5,148 &, 000 & 1,713 & 547 \\
\hline Standardisierter vorhergesagter Wert & $-3,671$ & 1,227 &, 000 & 1,000 & 547 \\
\hline Standardisierte Residuen & $-3,454$ & 3,003 &, 000 & ,999 & 547 \\
\hline
\end{tabular}

a. Abhängige Variable: Ihrer Lebensqualität 


\section{Streudiagramm}

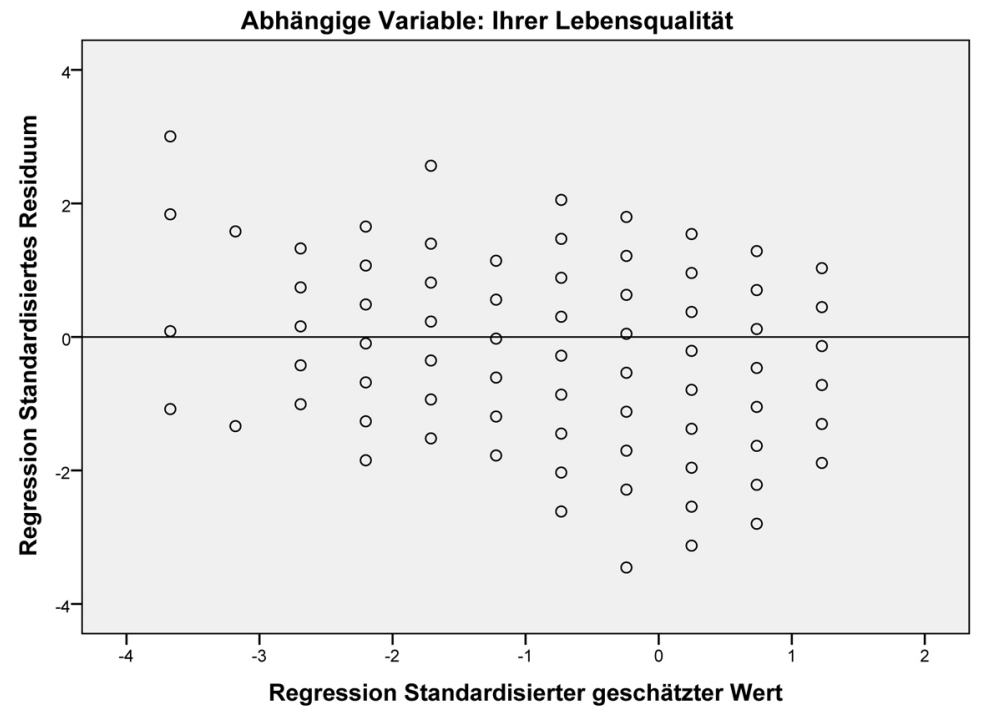

\subsection{Regression 2}

\begin{tabular}{|c|c|c|c|}
\hline \multicolumn{4}{|c|}{ Aufgenommene/Entfernte Variablen ${ }^{a}$} \\
\hline Modell & Aufgenommene Variablen & Entfernte Variablen & Methode \\
\hline \multirow[t]{11}{*}{1} & Soziale Integration, & . & Einschluss \\
\hline & Wie viele Kinder zwischen 6 und 18 Jahren leben in Ihrem Haushalt?, & & \\
\hline & Geschlecht, & & \\
\hline & Bildung, & & \\
\hline & Wie zufrieden sind Sie insgesamt mit Ihrer beruflichen Tätigkeit? Ziffer 0 = überhaupt nicht & & \\
\hline & zufrieden, 10 = sehr zufrieden, & & \\
\hline & Alter, & & \\
\hline & insgesamt die Umweltqualität in Ihrem Bundesland, & & \\
\hline & Wie schätzen Sie ganz allgemein Ihren Gesundheitszustand ein? $0=$ sehr schlecht, $10=$ aus- & & \\
\hline & gezeichnet, & & \\
\hline & Einkommen $^{\mathrm{b}}$ & & \\
\hline
\end{tabular}

a. Abhängige Variable: Ihrer Lebensqualität

b. Alle gewünschten Variablen wurden eingegeben.

\begin{tabular}{lccccc}
\hline \multicolumn{4}{c}{ Modellzusammenfassung $^{b}$} \\
\hline Modell & $\mathrm{R}$ & R-Quadrat & Korrigiertes R-Quadrat & Standardfehler des Schätzers & Durbin-Watson-Statistik \\
1 &, $622 \mathrm{a}$ &, 387 &, 372 & 1,514 & 1,889 \\
\hline
\end{tabular}

a. Einflussvariablen : (Konstante), Soziale Integration, Wie viele Kinder zwischen 6 und 18 Jahren leben in Ihrem Haushalt?, Geschlecht, Bildung, Wie zufrieden sind Sie insgesamt mit Ihrer beruflichen Tätigkeit? Ziffer o = überhaupt nicht zufrieden, $10=$ sehr zufrieden, Alter, insgesamt die Umweltqualität in Ihrem Bundesland, Wie schätzen Sie ganz allgemein Ihren Gesundheitszustand ein? o = sehr schlecht, 10 $=$ ausgezeichnet, Einkommen

b. Abhängige Variable: Ihrer Lebensqualität 
Hofbauer, Schwingsmehl: Bedeutet hohe Arbeitszufriedenheit, dass die Arbeitsbedingungen gut sind?

\begin{tabular}{llccccc}
\hline \multicolumn{7}{c}{ ANOVA $^{\text {a }}$} \\
\hline Modell & & Quadratsumme & df & Mittel der Quadrate & F & Sig \\
1 & Regression & 541,401 & 9 & 60,156 & 26,245 &, $000 \mathrm{~b}$ \\
& Nicht standardisierte Residuen & 857,224 & 374 & 2,292 & & \\
& Gesamt & 1398,625 & 383 & & & \\
\hline
\end{tabular}

a. Abhängige Variable: Ihrer Lebensqualität

b. Einflussvariablen : (Konstante), Soziale Integration, Wie viele Kinder zwischen 6 und 18 Jahren leben in Ihrem Haushalt?, Geschlecht, Bildung, Wie zufrieden sind Sie insgesamt mit Ihrer beruflichen Tätigkeit? Ziffer o = überhaupt nicht zufrieden, $10=$ sehr zufrieden., Alter, insgesamt die Umweltqualität in Ihrem Bundesland, Wie schätzen Sie ganz allgemein Ihren Gesundheitszustand ein? o = sehr schlecht, 10 = ausgezeichnet, Einkommen

\begin{tabular}{|c|c|c|c|c|c|c|c|c|}
\hline \multicolumn{9}{|c|}{ Koeffizienten $^{a}$} \\
\hline \multirow[t]{3}{*}{ Modell } & & \multirow{2}{*}{\multicolumn{2}{|c|}{$\begin{array}{l}\text { Nicht standardisierte } \\
\text { Koeffizienten }\end{array}$}} & \multirow{2}{*}{\multicolumn{2}{|c|}{$\begin{array}{l}\text { Standardisierte } \\
\text { Koeffizienten }\end{array}$}} & & \multirow{2}{*}{\multicolumn{2}{|c|}{ Kollinearitätsstatistik }} \\
\hline & & & & & & & & \\
\hline & & $\begin{array}{l}\text { Regressions- } \\
\text { koeffizientB }\end{array}$ & $\begin{array}{l}\text { Standard- } \\
\text { fehler }\end{array}$ & Beta & $\mathrm{T}$ & Sig. & Toleranz & VIF \\
\hline \multirow[t]{10}{*}{1} & (Konstante) &, 554 & ,665 & &, 833 &, 405 & & \\
\hline & $\begin{array}{l}\text { Wie zufrieden sind Sie insgesamt mit } \\
\text { Ihrer beruflichen Tätigkeit? Ziffer } 0= \\
\text { überhaupt nicht zufrieden, } 10=\mathrm{sehr} \\
\text { zufrieden. }\end{array}$ & ,371 & ,043 & ,391 & 8,689 &, 000 & 811 & 1,233 \\
\hline & Geschlecht & ,232 & 157 & ,061 & 1,482 & 139 & 972 & 1,029 \\
\hline & Alter & 259 & , 173 &, 063 & 1,496 & 135 & ,933 & 1,071 \\
\hline & Einkommen & ,685 & ,284 & 179 & 2,412 &, 016 & 297 & 3,361 \\
\hline & Bildung & 166 & ,289 & ,043 &, 573 &, 567 & 290 & 3,450 \\
\hline & $\begin{array}{l}\text { insgesamt die Umweltqualität in } \\
\text { Ihrem Bundesland }\end{array}$ & 159 &, 052 & 132 & 3,053 & ,002 & 871 & 1,148 \\
\hline & $\begin{array}{l}\text { Wie schätzen Sie ganz allgemein Ihren } \\
\text { Gesundheitszustand ein? } 0=\text { sehr } \\
\text { schlecht, } 10=\text { ausgezeichnet. }\end{array}$ & 220 & 048 & ,209 & 4,618 &, 000 & 802 & 1,247 \\
\hline & $\begin{array}{l}\text { Wie viele Kinder zwischen } 6 \text { und } 18 \\
\text { Jahren leben in Ihrem Haushalt? }\end{array}$ &,- 153 & ,092 &,- 069 & $-1,669$ & ,096 & 970 & 1,031 \\
\hline & Soziale Integration & ,399 &, 551 & ,030 & ,726 & ,469 & ,965 & 1,036 \\
\hline
\end{tabular}

\section{Residuenstatistik $^{\mathrm{a}}$}

\begin{tabular}{lllll}
\hline & Minimum & Maximum & Mittelwert & Standardabweichung N \\
Nicht standardisierter vorhergesagter Wert & 2,47 & 9,63 & 7,16 & 1,189 \\
Nicht standardisierte Residuen & $-6,141$ & 3,767 &, 000 & 1,496 \\
Standardisierter vorhergesagter Wert & $-3,943$ & 2,081 &, 000 & 1,000 \\
Standardisierte Residuen & $-4,056$ & 2,488 &, 000 & 384 \\
\hline
\end{tabular}

a. Abhängige Variable: Ihrer Lebensqualität 


\section{Streudiagramm}

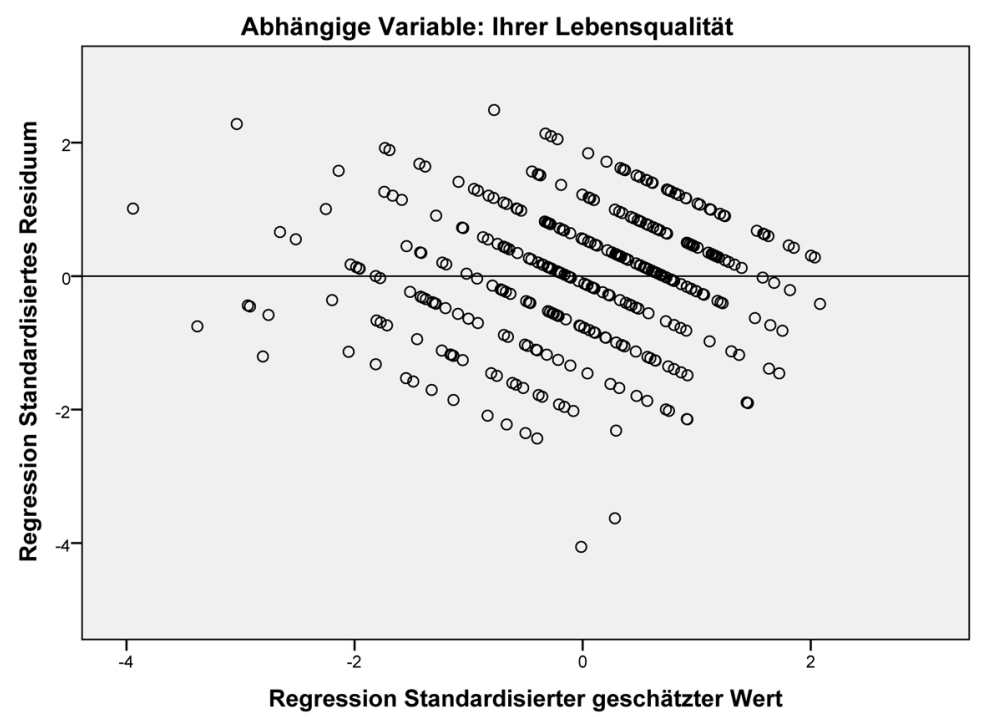

\subsection{Regression 3}

\begin{tabular}{llll}
\hline \multicolumn{2}{c}{ Aufgenommene/Entfernte Variablen $^{\text {a }}$} & \\
\hline Modell & Aufgenommene Variablen & Entfernte Variablen & Methode \\
1 & Bildung, Geschlecht, Faktor1 für Fragen aus 11 Aspekte der AZ, Faktor2 für Fragen aus 11 & . & Einschluß \\
\hline & Aspekte der AZ, Alter, Faktor3 für Fragen aus 11 Aspekte der AZ, Einkommenb & \\
\hline
\end{tabular}

a. Abhängige Variable: Wie zufrieden sind Sie insgesamt mit Ihrer beruflichen Tätigkeit? Ziffer o = überhaupt nicht zufrieden, $10=$ sehr zufrieden.

b. Alle gewünschten Variablen wurden eingegeben.

\begin{tabular}{lccccc}
\hline \multicolumn{5}{c}{ Modellzusammenfassung $^{{ }^{\prime}}$} \\
\hline Modell & $\mathrm{R}$ & R-Quadrat & Korrigiertes R-Quadrat & Standardfehler des Schätzers & Durbin-Watson-Statistik \\
1 &, $776 \mathrm{a}$ &, 602 &, 594 & 1,288 & 2,075 \\
\hline
\end{tabular}

a. Einflussvariablen : (Konstante), Bildung, Geschlecht, Faktorı für Fragen aus 11 Aspekte der AZ, Faktor2 für Fragen aus 11 Aspekte der AZ, Alter, Faktorз für Fragen aus 11 Aspekte der AZ, Einkommen

b. Abhängige Variable: Wie zufrieden sind Sie insgesamt mit Ihrer beruflichen Tätigkeit? Ziffer o = überhaupt nicht zufrieden, $10=\operatorname{sehr}$ zufrieden.

\begin{tabular}{|c|c|c|c|c|c|c|}
\hline \multicolumn{7}{|c|}{ ANOVA $^{a}$} \\
\hline Modell & & Quadratsumme & $d f$ & Mittel der Quadrate & $\mathrm{F}$ & Sig. \\
\hline \multirow[t]{3}{*}{1} & Regression & 840,797 & 7 & 120,114 & 72,434 &, $000^{\mathrm{b}}$ \\
\hline & Nicht standardisierte Residuen & 555,518 & 335 & 1,658 & & \\
\hline & Gesamt & 1396,315 & 342 & & & \\
\hline
\end{tabular}

a. Abhängige Variable: Wie zufrieden sind Sie insgesamt mit Ihrer beruflichen Tätigkeit? Ziffer $\mathrm{o}=$ überhaupt nicht zufrieden, $10=\operatorname{sehr}$ zufrieden.

b. Einflussvariablen : (Konstante), Bildung, Geschlecht, Faktorı für Fragen aus 11 Aspekte der AZ, Faktor2 für Fragen aus 11 Aspekte der AZ, Alter, Faktor3 für Fragen aus 11 Aspekte der AZ, Einkommen 


\begin{tabular}{|c|c|c|c|c|c|c|c|c|}
\hline \multicolumn{9}{|c|}{ Koeffizienten ${ }^{a}$} \\
\hline \multirow[t]{2}{*}{ Mode } & & \multicolumn{2}{|c|}{$\begin{array}{l}\text { Nicht standardisierte } \\
\text { Koeffizienten }\end{array}$} & \multicolumn{2}{|c|}{ Standardisierte Koeffizienten } & & \multicolumn{2}{|c|}{ Kollinearitätsstatistik } \\
\hline & & $\begin{array}{l}\text { Regressions- } \\
\text { koeffizientB }\end{array}$ & $\begin{array}{l}\text { Standard- } \\
\text { fehler }\end{array}$ & Beta & $\mathrm{T}$ & Sig. & Toleranz & VIF \\
\hline \multirow[t]{8}{*}{1} & (Konstante) & 7,491 & ,136 & & 55,208 &, 000 & & \\
\hline & $\begin{array}{l}\text { Faktor1 für Fragen aus } 11 \\
\text { Aspekte der AZ }\end{array}$ & 1,210 & 070 &, 599 & 17,285 &, 000 & ,988 & 1,013 \\
\hline & $\begin{array}{l}\text { Faktor2 für Fragen aus } 11 \\
\text { Aspekte der AZ }\end{array}$ & 917 & ,070 & ,455 & 13,035 &, 000 & ,975 & 1,026 \\
\hline & $\begin{array}{l}\text { Faktor3 für Fragen aus } 11 \\
\text { Aspekte der AZ }\end{array}$ & 421 & 078 & ,195 & 5,408 &, 000 & 914 & 1,094 \\
\hline & Geschlecht &,- 131 & 141 &,- 032 &,- 926 &, 355 &, 973 & 1,028 \\
\hline & Alter & ,039 & ,157 &, 009 & ,251 & 802 & 927 & 1,079 \\
\hline & Einkommen &,- 453 & 249 &,- 112 & $-1,817$ & ,070 & 313 & 3,196 \\
\hline & Bildung & ,312 & 254 & 077 & 1,227 & 221 & ,305 & 3,283 \\
\hline
\end{tabular}

a. Abhängige Variable: Wie zufrieden sind Sie insgesamt mit Ihrer beruflichen Tätigkeit? Ziffer o = überhaupt nicht zufrieden, $10=$ sehr zufrieden.

\begin{tabular}{lccccc}
\hline \multicolumn{7}{c}{ Residuenstatistik $^{\mathbf{a}}$} & & & \\
\hline & Minimum & Maximum & Mittelwert & Standardabweichung & $\mathrm{N}$ \\
Nicht standardisierter vorhergesagter Wert & 2,61 & 10,37 & 7,35 & 1,568 & 343 \\
Nicht standardisierte Residuen & $-4,926$ & 3,648 &, 000 & 1,274 & 343 \\
Standardisierter vorhergesagter Wert & $-3,025$ & 1,923 &, 000 & 1,000 & 343 \\
Standardisierte Residuen & $-3,825$ & 2,833 &, 000 &, 990 & 343 \\
\hline
\end{tabular}

a. Abhängige Variable: Wie zufrieden sind Sie insgesamt mit Ihrer beruflichen Tätigkeit? Ziffer o = überhaupt nicht zufrieden, $10=$ sehr zufrieden.

\section{Streudiagramm}

Abhängige Variable: Wie zufrieden sind Sie insgesamt mit Ihrer beruflichen

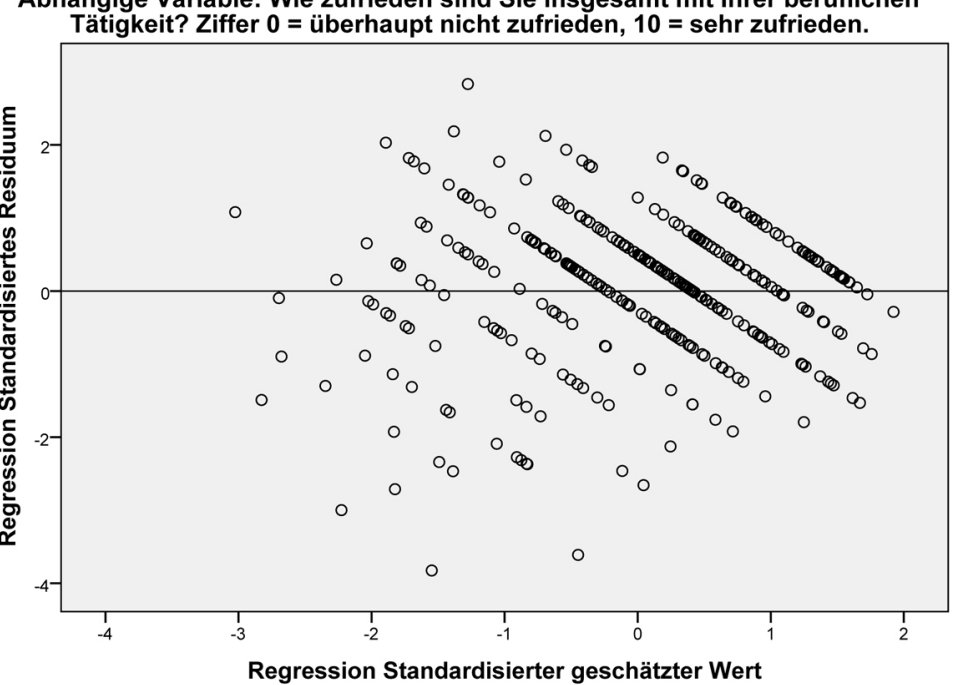




\section{Literatur}

Abele, A./Cohrs, J./Dette, D. (2006): Arbeitszufriedenheit - Person oder Situation? In: Fischer, L. (Hg.): Arbeitszufriedenheit. Konzepte und empirische Befunde. Göttingen: Hogrefe, 205-225.

Statistik Austria (2014): Wie geht's Österreich? - Indikatoren und Analysen. Wien: Statistik Austria.

Baethge, M. (1991): Arbeit, Vergesellschaftung, Identität. Zur zunehmenden normativen Subjektivierung der Arbeit. Soziale Welt, 42 (1), 6-19.

Bakker, A./Demerouti, E. (2007) "The Job Demands-Resources model: state of the art", Journal of Managerial Psychology, 22 (3) 309-328, URL http://www.emeraldinsight.com/doi/full/10.1108/02683940710733115 [15.5.2017]

Boarini, R./Comola, M./Smith, C./Manchin, R./De Keulenaer, F. (2012): What Makes for a Better Life? The Determinants of Subjective Well-Being in OECD Countries - Evidence from the Gallup World. OECD Statistics Working Papers, No. 2012/03, OECD Publishing. http://dx.doi. org/10.1787/5kgb9ltjm937-en [12.2.2017]

Bolder, A./Naevecke, S./Schulte, S. (2005): Türöffner Zeitarbeit: Kompetenz und Erwerbsverlauf in der Praxis der Leiharbeit. Wiesbaden: VS Verlag für Sozialwissenschaften.

Brenke, K. (2015): Arbeitszufriedenheit. Die große Mehrzahl der Beschäftigten in Deutschland ist mit ihrer Arbeit zufrieden. DIW Wochenbericht, 32/33.

Bruggemann, A. (1976): Zur empirischen Untersuchung verschiedener Formen von Arbeitszufriedenheit. Zeitschrift für Arbeitswissenschaft, 30 (2), 71-74.

Diener, E. (1999): Personality and subjective well-being. In: Kahneman, D./Diener, E./Schwarz, N. (Hg.): Well-being: The foundations of hedonic psychology. New York: Russel Sage foundation, 213-229.

Diener, E./Eunkoook, M./Suh, R./Robert, E./Smith, H. (1999): Subjective Well-being: Three decades of progress. Psychological Bulletin, 125 (2), 276-302.

Eid, M./Larsen, R. (2008): Ed Diener and the Science of Subjective Well-being. In: Eid, M./Larsen, R.: The Science of Subjective Well-being. New York/London: Guilford Press, $1-17$.

Eiffe, F./Ponocny, I./Gärtner, K./Till, M. (2016): Analytical report on subjective Well-being. 2016 edition. Luxembourg: European Union.

Eurofound (2012): Trends in job quality in Europe. A report based on the fifth European Working Conditions Survey. Luxembourg: European Foundation for the Improvement of Living and Working Conditions.

Eurofound (2014): European Foundation for the Improvement of Living and Working Conditions. Online: https://www. eurofound.europa.eu/sites/default/files/tn1405020s.pdf [11. 11. 2015].

Festinger, L. (1957): A Theory of cognitive Dissonance. California: Stanford University Press
Fietze, S. (2011): Arbeitszufriedenheit und Persönlichkeit: „Wer schaffen will, muss fröhlich sein!“. Berlin: SOEPpapers. https://www.diw.de/documents/publikationen/73/ diw_01.c.376207.de/diw_spo388.pdf [12.1.2017]

Fleche, S./Smith, C./Sorsa, P. (2011): Exploring Determinants of Subjective Wellbeing in OECD Countries: Evidence from the World Value Survey. OECD Economics Department Working Papers, Nr. 921, OECD Publishing. http://www. oecd-ilibrary.org/docserver/download/5kgok6zlcm $5 \mathrm{k}$ en.pdf? expires $=1492675549 \& \mathrm{id}=\mathrm{id} \& \mathrm{accname}=$ guest $\&$ checksum $=50 \mathrm{~A} 6 \mathrm{C}_{3} 615300 \mathrm{EFC} 6 \mathrm{C}_{5} \mathrm{CBB}_{2} \mathrm{C}_{3} \mathrm{EAD} 492 \mathrm{E}$ [27.1.2017]

Graham, C. (2011): The Pursuit of Happiness. Washington, D.C.: Brookings Institution Press.

Green, F. (2006): Demanding work: The paradox of jobquality in the affluent economy. Princeton: University Press.

Hardering, F. (2011): Sicherheit macht zufrieden. www.fortschrittszentrum.de: Zentrum für gesellschaftlichen Fortschritt. http://www.fortschrittszentrum.de/dokumente/2011-09_Sicherheit_macht_zufrieden.pdf [26.9.2015]

Hofinger, C. (2009): Meine Situation könnte noch schlechter sein. Eine neue Skala zur Messung Resignativer Arbeitszufriedenheit. In: Kammer für Arbeiter und Angestellte für OÖ (Hg.): Schriftenreihe Österreichische Arbeitsklimaindex I. Linz, 46-53. https://media.arbeiterkammer. at/ooe/publikationen/arbeitsklimaindex/AKI_Schriftenreihe_Band_1.pdf [2.2.2015]

Holtgrewe, U. (2013): Qualität der Arbeit in Europa: Vergleichen, aber was? Trendreport, 2, 4-5. http://www.forba.at/ data/downloads/file/821-Trendreport_2-2013_Webversion.pdf, [21.12.2016]

Hölzl, E. (2011): Kapitel II: Arbeitsgestaltung. In Kirchler, E. (Hg.) Arbeits- und Organistationspsychologie. 3. Auflage. Wien: facultas.wuv, 199-318.

Inglehart, R. (1977): The silent revolution. Princeton. Princeton University Press

Kahneman, D./Deaton, A. (2010): High income improves evaluation of life but not emotional well-being. Proceedings of the National Academy of Sciences, 107 (38), 10.1073/pnas.1011492107.

Kahneman, D./Krueger, A./Schkade, D. A./Schwarz, N./ Stone, A. (2004): A survey method für characterizing daily life experience: The Day Reconstruction Method. Science, 306 (5702), 1176-1780.

Legatum Institute (2015): www.prosperity.com. http:// www.prosperity.com/download_file/view_inline/2833 [18.6.2015]

Lengfeld, H./Hirschle, J. (2009): Die Angst der Mittelschichten vor dem sozialen Abstieg. Zeitschrift für Soziologie, 5, 379-398.

Marrs, K. (2010): Herrschaft und Kontrolle in der Arbeit. In: Böhle, F./Voß, G./Wachtler, G. (Hg.): Handbuch Arbeitssoziologie. Wiesbaden: VS Verlag für Sozialwissenschaften, 331-358. 
Metzger, E./Vermeylen, G./Parent-Thirion, A. (2011): Arbeits- und Beschäftigungsqualität im European Working Conditions Survey. WISO, 34 (Sonderheft), 183-198.

OECD (2015): http://www.oecdbetterlifeindex.org/de/media/ bli/documents/BLIZUSAMMENFASSUNG_2015.pdf [17.1.2017]

Poggi, A./Villosio, C. (2015): Subjective Well-Being at the workplace. In: Holtgreve, U./Kirov, V./Ramioul, M.: Hard Work in New Jobs. New York: Palgrave Macmillan, $70-83$.

Raml, R. (2012): Eine theoretische Evaluierung des Arbeitsklima Index. Schriftenreihe Österreichischer Arbeitsklima Index 1. https://media.arbeiterkammer.at/ooe/publikationen/arbeitsklimaindex/AKI_1_Raml_Eine_theoretische_Evaluierung.pdf [9.1.2017]

Sigrist, J. (2000): A Theory of Occupational Stress. In: Dunham, J.: Stress in the Workplace. London: Whurr Publishers, 63-66.

Six, B./Felfe, J. (2004): Einstellungen und Werthaltungen im organisationalen Kontext. In: Schuler, H. (Hg.): Organisationspsychologie 1 - Grundlagen und Personalpsychologie. Enzyklopädie der Psychologie, Bd. D/III/3. Göttingen: Hogrefe, 597-672.

Van Saane, N./Sluiter, J./Verbeek, J./Frings-Dresen, M. (2003): Reliability and validity of instruments measuring job satisfaction - a systematic review. Occupational Medicine, 53 (3), 191-200.

Wanous, J. P./Reichers, A./Hudy, M. J. (1997): Overall job satisfaction: how good are single-item measures? Journal of applied Psychology, 82 (2), 247-252.

Zapf, W. (1972): Zur Messung der Lebensqualität. Zeitschrift für Soziologie, 1 (4), 353-376. 